\title{
Assiste-t-on à une transformation uniforme des carrières professionnelles vers plus de mobilité ?
}

Une exploitation de l'enquête Histoire de vie

General Increase in mobility on French labour market? The data of the French survey called Histoire de vie

Guillemette de Larquier et Delphine Remillon

\section{OpenEdition}

Journals

Édition électronique

URL : http://journals.openedition.org/travailemploi/4082

DOI : 10.4000/travailemploi.4082

ISSN : 1775-416X

Éditeur

DARES - Ministère du Travail

Édition imprimée

Date de publication : 17 mars 2008

Pagination : 13-30

ISSN : 0224-4365

\section{Référence électronique}

Guillemette de Larquier et Delphine Remillon, « Assiste-t-on à une transformation uniforme des carrières professionnelles vers plus de mobilité ? », Travail et Emploi [En ligne], 113 | janvier-avril 2008, mis en ligne le 17 février 2010, consulté le 19 avril 2019. URL : http://journals.openedition.org/ travailemploi/4082 ; DOI : 10.4000/travailemploi.4082 


\title{
Assiste-t-on à une transformation uniforme des carrières professionnelles vers plus de mobilité? Une exploitation de l'enquête "Histoire de vie "
}

\author{
Guillemette de Larquier(*), Delphine Remillon (**)
}

\begin{abstract}
En exploitant une base originale de 4508 carrières sur les quarante dernières années, extraite de l'enquête "Histoire de vie» de l'Insee, les auteures mettent en question le constat, assez partagé mais également controversé, d'une transformation profonde du marché du travail dans le sens d'un affaiblissement des marchés internes et d'une mobilité de carrière accrue. Leur enquête apporte une réponse mitigée; elles concluent en effet à la permanence d'un schéma traditionnel des carrières stables - avec, toutes choses égales par ailleurs, une diminution pour les hommes des changements professionnels en début de carrière. Cette stabilité se constate par contre dans un environnement de plus grande insécurité professionnelle due à la hausse du chômage et à des transitions emploichômage qui sont en augmentation pour toutes les générations mais non de manière uniforme. Les auteures concluent à une stabilité relative de la mobilité au sein de l'emploi et à une hausse des mouvements avec le chômage. La nouvelle donne des carrières semblerait davantage être le risque de chômage que l'apparition d'un modèle de mobilité générale.
\end{abstract}

Permettre aux individus de concilier flexibilité du travail et sécurité des carrières, tel semble être le nouveau mot d'ordre de la politique de l'emploi en Europe. Derrière cette idée commune traduite par le néologisme de «flexicurité» par les organismes internationaux (CONSEIL EUROPÉEN, 2003; OCDE, 2004), des perspectives très diverses sont exprimées, au point qu'un rapport et une organisation syndicale, aux visées guère conciliables, peuvent reprendre la même expression de «sécurité sociale professionnelle» (Cahuc, Kramarz, 2004; CGT, 2006). Au-delà des divergences, ces travaux se rejoignent sur le présupposé d'une transformation profonde du marché du travail, dans le sens d'un affaiblissement des marchés internes et d'une mobilité des carrières accrue. Or, ce constat est controversé; les travaux existant sur l'évolution de la mobilité professionnelle sont loin d'être unanimes et défendent même des points de vue divergents que GERME (2001) et RAMAuX (2006) présentent comme deux thèses opposées. D'un côté, certains défendent l'idée d'une érosion des marchés internes et d'une flexibilisation progressive et générale de l'emploi; les autres affirment que seule la précarité s'est développée laissant les marchés

(*) Economix (UMR 7166) et Centre d'études de l'emploi; larquier@u-paris10.fr

(**) Economix (UMR 7166) et Centre d'études de l'emploi; delphine.remillon@u-paris10.fr internes relativement inchangés mais accentuant le dualisme du marché du travail. Cet article s'inscrit dans ces débats empiriques en posant précisément la question de l'augmentation des mobilités professionnelles dans les carrières et en s'interrogeant sur le caractère uniforme ou non de ces évolutions.

Il existe en France une longue tradition d'étude de la mobilité sociale - essentiellement des promotions - dominante dans les années 1960 et jusqu'au début des années 1980. Mais avec la montée du chômage, les mobilités involontaires se sont accrues et il est apparu nécessaire de distinguer plus nettement mobilité professionnelle et mobilité sociale et de trouver de nouvelles mesures de la mobilité professionnelle (Coutrot, Dubar, 1992). Or, à travers les différentes sources existantes, il est relativement difficile de reconstituer les carrières complètes des individus sur le marché du travail. Si les résultats des travaux sur la mobilité sont si peu convergents, c'est justement parce qu'ils portent sur des sources, des périodes et des sous-populations différentes. Généralement, les travaux traitant de la mobilité sur le marché du travail français utilisent quatre types d'enquêtes. L'enquête FQP (Enquête sur la formation et la qualification professionnelle) est la source clef sur la mobilité sociale en France (INSEE, 2006). Elle permet également d'appréhender l'évolution de la mobilité professionnelle (Chapoulie, 2000), grâce à des données précises 
sur les changements d'établissements, d'entreprises et de PCS, recueillies à intervalles réguliers (six enquêtes depuis 1964). Mais l'information est parcellaire puisqu'on ne saisit la situation des personnes interrogées qu'à trois moments de leur vie professionnelle: la date de l'enquête, cinq ans auparavant, et au début de leur vie professionnelle, en ignorant les événements qui se sont déroulés dans l'intervalle. L'enquête Emploi n'a pas pour objet principal l'étude de la mobilité professionnelle mais constitue une mesure régulière de l'emploi et permet le décompte des chômeurs. Elle peut néanmoins être utilisée pour étudier l'évolution de la mobilité sur courte période (AMOSSÉ, 2003) et surtout, à deux reprises, ont été menées sur un sous-échantillon des enquêtes complémentaires, portant plus spécifiquement sur la question des mobilités (Goux, 2003): l'enquête Jeunes et carrières (1997) et Carrière et mobilité (1989). Les enquêtes d'insertion développées par le Cereq, et plus particulièrement les trois enquêtes "Génération » permettent une analyse fine de la mobilité professionnelle des jeunes sur environ trois ans, voire sept ans pour l'enquête 1998 (pour laquelle il est même prévu une réinterrogation à dix ans), mais elles ne vont pas au-delà des premières années de carrière et sont confrontées à d'importants problèmes d'attrition de l'échantillon. Enfin, les DADS constituent une autre source possible de l'analyse des mobilités (Leclair, Roux, 2005; Delarre, Duhautois, 2004). Ces données d'origine administrative ont l'avantage d'être exhaustives et de fournir une information très détaillée sur la durée d'emploi, l'entreprise et la rémunération non sujette aux biais de déclaration ou de mémoire (contrairement aux trois premières sources) mais la principale limite est qu'il est impossible de suivre les salariés d'un emploi à l'autre ou vers le chômage.

Toutes ces sources fournissent donc une information partielle sur les mobilités, pour différentes périodes, et différentes sous-populations. Elles ne permettent pas de reconstituer l'ensemble de la carrière des individus sur le marché du travail (soit les panels ne sont pas conduits sur des périodes assez longues, soit il y a des problèmes d'attrition de l'échantillon) rendant ainsi «difficile le repérage des principales évolutions de ces trente dernières années » (GERME, 2001). Pourtant, il semble que ce soit la durée minimum, ajoute Germe, pour apprécier l'évolution de la mobilité, car les facteurs de transformation évoqués (érosion des marchés internes, changements de l'organisation du travail) sont forcément lents. Dans l'enquête Histoire de vie - Construction des identités (2003) exploitée ici, ont été collectées des données biographiques qui permettent de reconstituer de telles trajectoires ( $c f$. encadré 1); en contrepartie, nous ne disposons pas d'une information représentative du marché du travail sur l'ensemble de la période. Ainsi, nous n'étudions pas le marché du travail français et son évolution dans le temps, mais des trajectoires professionnelles qui se sont déroulées en son sein, la plus ancienne de notre échantillon commençant en 1953. Depuis les travaux fondateurs de l'Ined autour de l'enquête dite « $3 \mathrm{~B}$ », les enquêtes biographiques se sont développées, surtout en démographie (Courgeau, LeliÈvre, 1990) et l'on dispose désormais de plusieurs grandes enquêtes de ce type (la dernière en date étant l'enquête Familles et employeurs, 2005) qui fournissent des données très intéressantes sur longue période, même si elles n'en sont pas exemptes de biais ( $c f$. encadré 1$)$. Nous proposons de mobiliser cette source originale dans l'étude de l'évolution des mobilités dans les carrières, vues comme l'ensemble des transitions emploi-emploi et emploi-chômage. L'analyse est restreinte aux transitions au sein de l'activité: nous ne nous intéressons ni à l'insertion dans l'emploi(1) (déjà largement étudiée dans les travaux du Cereq), ni aux sorties vers la retraite. Au final, la population analysée est composée de 4508 individus (trois générations nées entre 1940 et 1969) ayant tous eu une activité professionnelle sur le marché du travail français d'au moins un an(2). Dans une première partie, les carrières de trois générations sont décrites en construisant trois indicateurs de mobilité. Nous poursuivons par une série de tests de corrélations "toutes choses égales par ailleurs» nous permettant de préciser notre diagnostic (analyse de la mobilité à conjoncture et structure de la population contrôlées) et de voir quelles sont les catégories de population les plus mobiles.

\section{La mobilité, nouvelle donne des carrières?}

Nous décrivons ici l'évolution des carrières en termes de mobilités: les trajectoires professionnelles sont-elles effectivement plus mobiles, moins linéaires? Et si oui, quels types de transitions professionnelles se sont accrus? L'opinion répandue aujourd'hui est qu'avec l'affaiblissement des marchés internes, les carrières se seraient radicalement transformées et que l'on serait désormais obligé de changer d'entreprise, de région (voire de pays), de métier, de statut plusieurs fois au cours de sa vie active; par ailleurs, l'insécurité aurait augmenté et représenterait désormais un risque pour tous. Néanmoins, au plan empirique, ces questions restent controversées, même concernant l'insécurité: FoUgère (2003) ou L'HorTy (2004) ne concluent pas à une hausse, tandis que GIVORD et Maurin (2003) ou Behaghel (2003) défendent la position inverse (3).

(1) En particulier, nous ne nous intéressons pas ici aux chômeurs n'ayant jamais travaillé.

(2) Voir la composition de l'échantillon en annexe.

(3) Pour un récapitulatif des études empiriques sur l'insécurité en France et une analyse des différences de résultats entre ceux qu'il nomme respectivement les «fixistes» et les «évolutionnistes », $c f$. L'HORTY (2004). 


\section{Encadré 1}

\section{La mesure des mobilités dans l'enquête Histoire de vie}

Histoire de vie est une enquête Insee inédite qui cherche à saisir le mode de construction des identités. Le questionnaire débute par une partie chronologique dans laquelle 8403 personnes de 18 ans et plus ont été invitées à reconstruire leur biographie familiale, résidentielle et professionnelle. C'est cette partie biographique que nous exploitons. II s'agit donc de données rétrospectives, qui décrivent, pour chaque individu, les transitions emploichômage et emploi-inactivité, ainsi que les transitions emploi-emploi codées selon trois types de changements:

- de statut (salarié de l'État, d'une collectivité locale, d'une entreprise publique, du secteur privé, de sa propre entreprise, et non salarié);

- de profession dans la nomenclature PCS 2003;

- de qualification (manœuvre; ouvrier qualifié; technicien; agent de maîtrise; cadre; employé), qualification telle que déclarée par l'enquêté, qui n'est pas toujours cohérente avec la qualification finalement retenue pour identifier la profession dans la nomenclature PCS.

La précision des données est annuelle. Si un individu a connu plusieurs états sur le marché du travail, au cours de la même année, c'est l'état principal qui est retenu. En particulier, cela signifie que seules les périodes «longues" de chômage sont repérées.

Le premier intérêt de ces données est qu'elles permettent de très longues fenêtres d'observation. Les carrières de la génération la plus ancienne de notre échantillon ont une durée moyenne de trente et un ans, celles de la génération la plus récente une durée moyenne de seize ans; c'est sans commune mesure avec les fenêtres d'observations habituelles des études sur les mobilités. Le second intérêt est la collecte des informations sur des cohortes, ce qui permet de distinguer les effets âge et date des effets génération. Troisième intérêt, cette méthode de collecte produit un questionnement homogène pour les différentes générations.

Deux limites cependant. Les changements d'employeur ne sont pas repérés en tant que tels, ce qui ne permet pas de distinguer les mobilités internes des mobilités externes (à l'entreprise)(1). Nous pouvons seulement distinguer les mobilités qui s'effectuent dans l'emploi (mobilités internes à l'emploi) de celles qui passent par une période de chômage ou d'inactivité (mobilités externes à l'emploi). Par ailleurs, du fait du caractère rétrospectif du questionnement, il existe des biais de mémoire et de sélection: biais de sélection, car seules les personnes encore vivantes et présentes en France en 2003 ont été interrogées. Pour limiter ce biais de mortalité différentielle selon les groupes sociaux, nous avons exclu de l'échantillon les générations les plus anciennes (celles nées avant 1940). En contrepartie, nous n'avons pas de problème d'attrition de l'échantillon. Les oublis et erreurs de datation peuvent également peser sur la qualité des informations recueillies. Cependant, s'inspirant de l'expérience du "groupe de réflexion sur l'approche biographique " le questionnaire a été conçu de façon à limiter au maximum ces biais, en demandant à l'enquêté de dater d'abord les événements familiaux, puis ses changements résidentiels, ce qui lui permet ensuite de situer plus facilement les événements professionnels, par rapport à ces premiers repères (Crenner et al., 2006). Auriat (1997) explique en effet qu'il est essentiel, dans une enquête biographique, de faire situer les différents événements les uns par rapport aux autres, un événement permettant de corriger la date de l'autre. Elle montre également que le type d'erreur temporelle n'est pas lié directement à la longueur de la période rétrospective mais au type d'événement, certains étant plus difficiles à se remémorer que d'autres. Enfin, la qualité de la mémorisation temporelle dépend du type d'événement concerné, certains événements ayant un rythme caractérisé par les mois, d'autres l'année, ce qui est le cas des changements d'emploi. Ici, on ne demande effectivement à la personne interrogée que de se remémorer les grandes étapes de sa vie professionnelle (il ne s'agit que des périodes supérieures à un an) c'est-à-dire les plus marquantes. Au total, le risque de sous-estimation des mobilités éloignées dans le passé n'est pas à négliger. II en sera tenu compte dans nos commentaires.

(1) Extrait du guide d'instructions aux enquêteurs: “Changer d'employeur n'équivaut pas forcément à changer de profession: on ne recueillera pas les changements d'employeurs en tant que tels, mais s'ils correspondent à un changement de statut, de position professionnelle ou de profession, ils seront matérialisés à ce titre. 》 (p. 16).

Il importe de distinguer les deux types de mobilités que sont l'instabilité et l'insécurité (CERC, 2005). La stabilité de l'emploi «désigne la continuité du lien d'emploi, entre un salarié et une entreprise». Les indicateurs utilisés pour mesurer cette stabilité/instabilité sont la durée totale passée dans l'entreprise ou l'ancienneté moyenne ou encore le taux de rotation de la main-d'œuvre. La sécurité, quant à elle, "désigne le fait, pour une personne, de demeurer employée, sans interruption durable, même s'il y a changement d'entreprise. » Deux indicateurs sont généralement proposés, le taux annuel de transition emploi-chômage ou le taux annuel de transition emploi-non emploi.

Dans notre enquête, la mesure de l'insécurité est possible. Nous limitons dans cette partie la définition de l'insécurité aux transitions emploi-chômage pour les salariés du secteur privé. Cette mesure n'est pas totalement satisfaisante car elle sous-estime les sorties involontaires du marché du travail, une part non négligeable de ces sorties s'effectuant vers l'inactivité. En outre, la méthode d'enquête rétrospective ne permet pas de départager les recherches 
d'emploi actives des recherches passives, comme on le fait habituellement dans l'enquête Emploi. Le classement entre inactivité et chômage est donc plus incertain. Mais ce choix nous permet d'avoir des résultats comparables avec les autres travaux cités. Avec néanmoins une limite à la comparabilité des résultats (et notamment celle des niveaux d'insécurité) : toutes les sorties vers le chômage de «courte» période nous échappent (l'année $t+1$ n'étant alors pas renseignée en chômage) ce qui conduit à sousestimer l'insécurité.

Nous ne pouvons, par contre, mesurer ni l'instabilité ni même la mobilité au sens d'Amossé(4) (2002), puisque le changement d'employeur n'est pas une information disponible. Il faut donc construire une autre mesure de la mobilité qui tienne compte également des mobilités volontaires(5), sans fonder cette mesure sur les changements d'entreprise. Nous proposons ainsi un nouvel indicateur, le taux de changement professionnel, qui décompte les changements de PCS 2003, c'est-à-dire les changements de professions avec ou sans changement de qualification ou de statut ( $c f$. détails dans l'encadré 1). Cet indicateur nous semble intéressant pour tester, sur longue période, l'idée d'une transformation des trajectoires professionnelles, dans le sens d'un accroissement des transitions, y compris internes à l'entreprise.

$\mathrm{Au}$ final, nous définissons la mobilité sur le marché du travail comme la somme des changements professionnels et des transitions entre emploi et chômage. Ces indicateurs sont calculés pour un seuil minimum de cent personnes.

\section{Une hausse de la mobilité...}

Le premier indicateur est celui de la mobilité totale sur le marché du travail, hors première entrée et sortie définitive (6). Il fournit une lecture globale des évolutions de la mobilité par génération, que celle-ci soit interne ou externe à l'entreprise et interne ou externe à l'emploi.

L'effet le plus marquant est l'effet cycle de vie, observé pour toutes les cohortes ( $c f$. graphique 1): après une première phase haute d'environ dix ans, la mobilité diminue avec l'âge. On remarque que les pics de la mobilité en début de carrière sont

(4) Il définit la mobilité professionnelle comme la somme des transitions suivantes: rester en emploi en changeant d'employeur, passer de l'emploi au chômage ou du chômage à l'emploi, être au chômage aux deux enquêtes en ayant connu entre-temps un emploi.

(5) Une autre façon de distinguer instabilité et insécurité (cf. Behaghel, 2003) consiste à définir l'insécurité comme la probabilité de départ involontaire du salarié, par opposition à l'instabilité de l'emploi, plus large, qui englobe à la fois les départs involontaires et volontaires.

(6) (changement professionnels + transitions chômage-emploi + transitions emploi-chômage) entre $t$ et $t+1$

individus en emploi + chômeurs (sauf ceux n'ayant jamais travaillé) en $t$ décalés de génération en génération. C'est particulièrement vrai pour la dernière génération: dans le graphique 1 , si l'on décale artificiellement la courbe [1960-69] d'un an vers la gauche (on rajeunit d'un an les individus), la courbe se superpose bien avec les deux premières tout en atteignant des niveaux légèrement plus élevés. Cela s'explique par un âge moyen d'entrée dans la vie active plus tardif (augmentation d'un an de l'âge moyen de fin d'études entre les deux générations extrêmes de notre échantillon, $c f$. annexe). En fin de carrière, la mobilité devient très faible: elle tombe à environ $3 \%$ pour les plus âgés de notre échantillon (les 55 ans et plus). Au-delà de cet effet âge, très stable dans le temps, on constate graphiquement une hausse de la mobilité de génération en génération. À y regarder de plus près, il semble que ce soit surtout la dernière génération [1960-69] qui se distingue des deux autres par une mobilité accrue. Néanmoins, cette hausse est limitée, le taux maximum de mobilité passant seulement de $12,7 \%$ pour la génération [1940-49] à 14,8\% pour la génération [1960-69], soit une augmentation d'un peu moins de deux points.

Le graphique 2, qui décrit la part des mobilités sans passage par le chômage selon l'âge pour les trois générations, montre que cette part est décroissante avec l'âge. Plus on avance dans sa carrière, plus la part des mobilités involontaires (liées au chômage) est importante. Mais l'effet qui domine est surtout un effet date: la part croissante des mobilités avec le chômage, de génération en génération (à âge donné) reflète la dégradation de l'état du marché du travail ayant entraîné l'apparition de chômage dans les carrières. Ainsi on voit que la génération la plus ancienne n'a eu quasiment aucune mobilité liée au chômage jusque vers ses $30-33$ ans, alors que la génération 1960-1969 a connu un contexte de chômage, dès son entrée sur le marché du travail.

À première vue, on observe donc une légère hausse de la mobilité, de génération en génération, avec un saut plus marqué concernant la dernière génération par rapport aux deux autres, et on pressent qu'une part importante de cette hausse est liée à l'augmentation du chômage. Pour mieux comprendre ce qu'il $\mathrm{y}$ a derrière cette hausse apparente de la mobilité, il est nécessaire de passer à l'étude détaillée des deux principales transitions sur le marché du travail: les changements professionnels et les transitions emploi-chômage. Pour les secondes, nous proposons des graphiques par année (et non par âge) car c'est l'effet conjoncturel qui prime (la dégradation du marché du travail à partir des années soixantedix) et non un effet cycle de vie.

\section{... due à une hausse des changements professionnels chez les femmes...}

Nous nous focalisons à présent sur les mobilités internes à l'emploi, c'est-à-dire sans période longue 
Graphique 1

\section{Taux de mobilité selon l'âge}

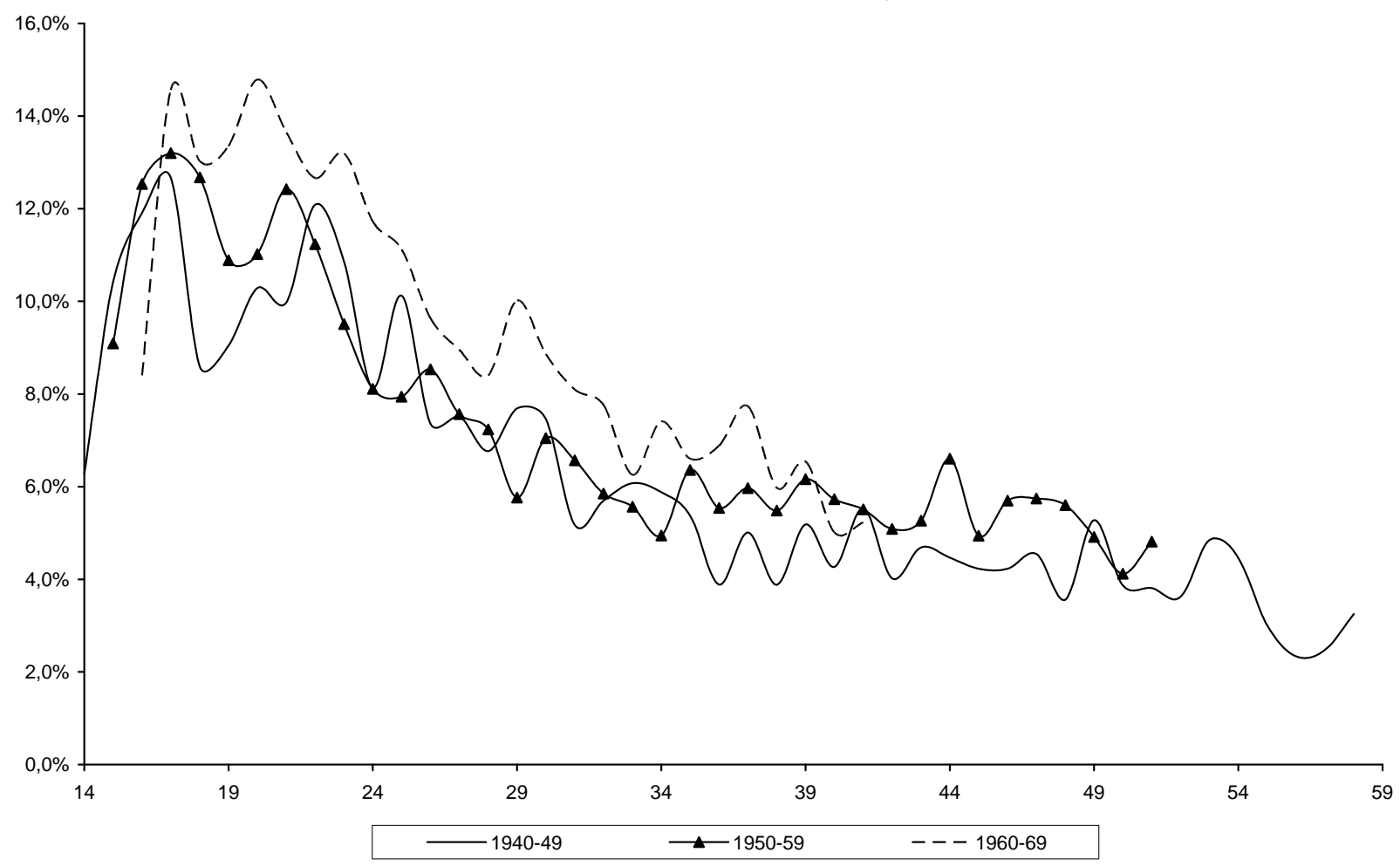

Indicateur: pourcentage des actifs (ayant déjà travaillé) d'une génération donnée qui ont connu une mobilité entre les années $t$ et $t+1$. Champ: emploi total + chômeurs (sauf ceux n'ayant jamais travaillé).

Source: Enquête Histoire de vie, Insee, 2003.

Graphique 2

Part des mobilités sans passage par le chômage selon l'âge

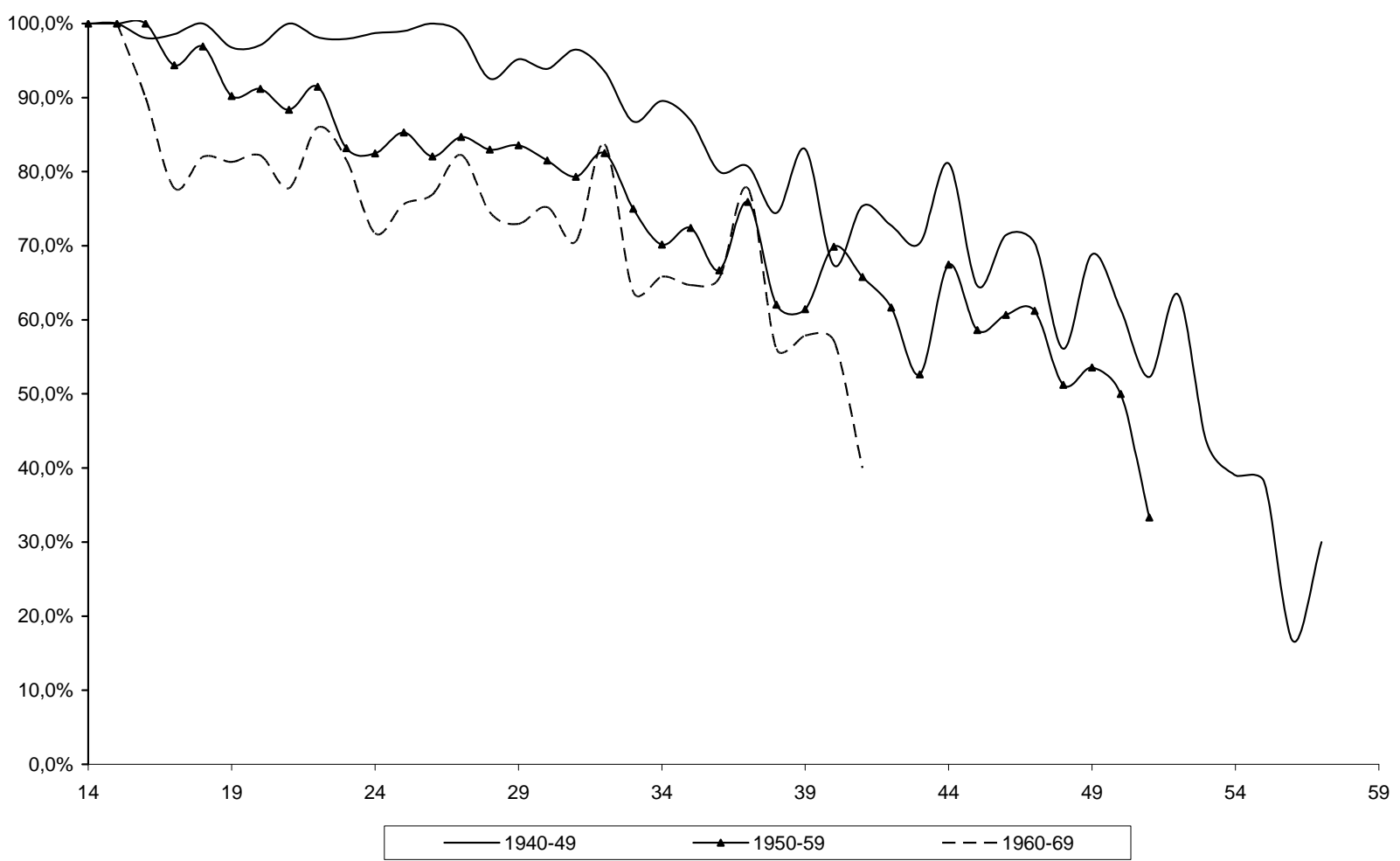

Indicateur: parmi les actifs mobiles entre les années $t$ et $t+1$, pourcentage des individus qui ont connu une mobilité sans passage par le chômage.

Champ: ensemble des mobilités entre $t$ et $t+1$.

Source: Enquête Histoire de vie, Insee, 2003. 
Graphique $3 \mathrm{a}$

Taux de changement professionnel des hommes selon l'âge

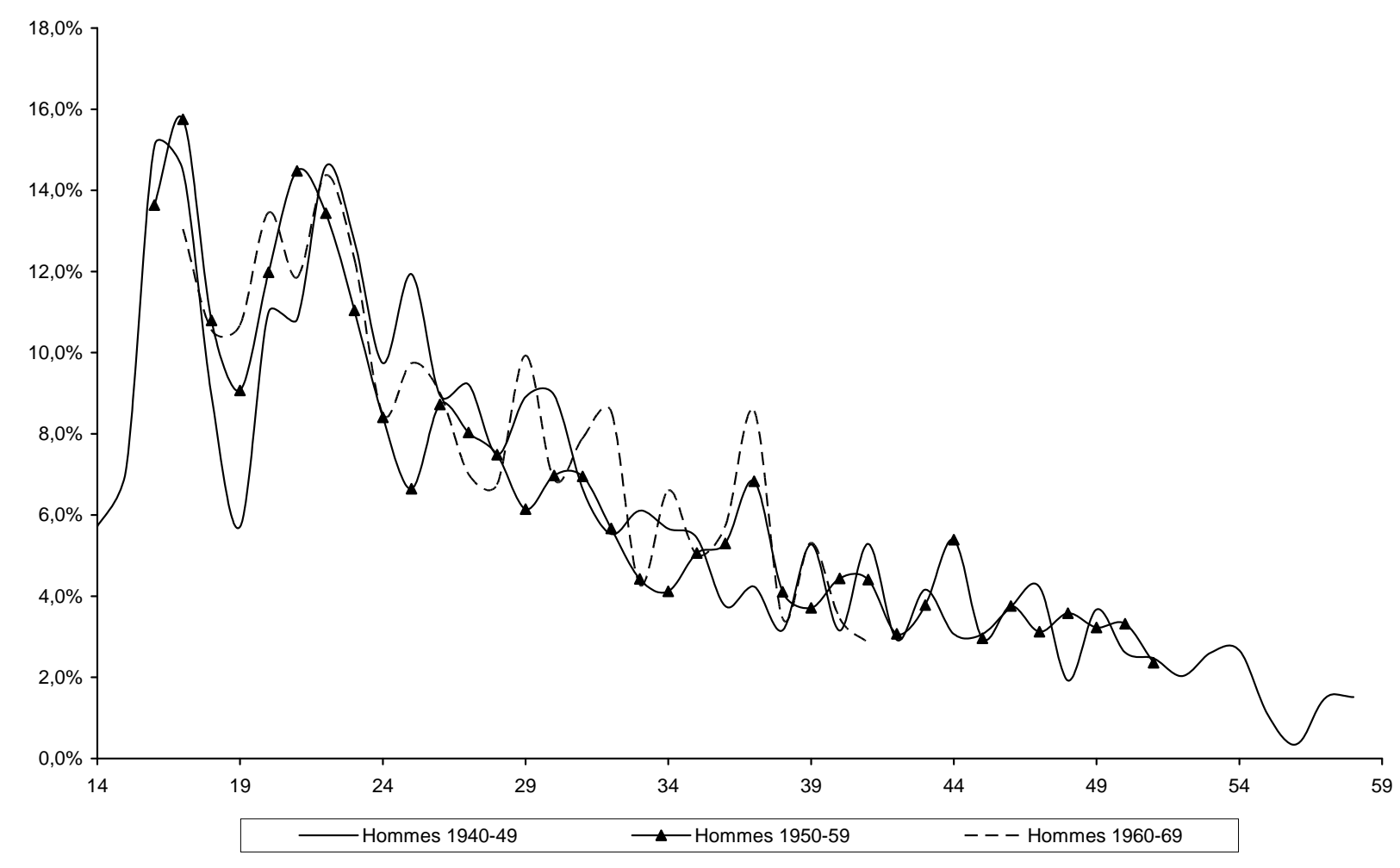

Graphique 3b

Taux de changement professionnel des femmes selon l'âge

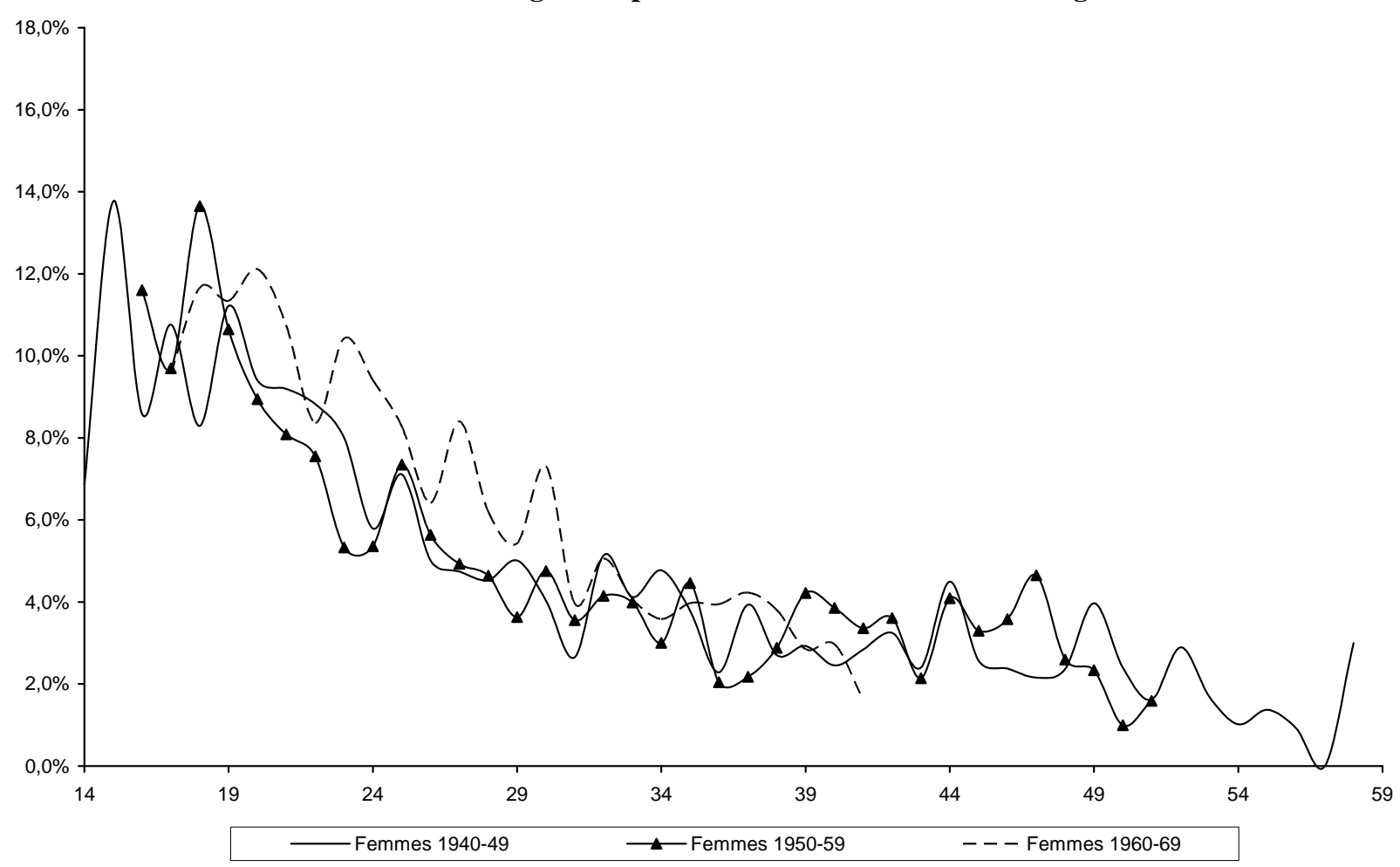

Indicateur: pourcentage des actifs occupés d'une génération donnée, qui ont connu un changement professionnel entre $t$ et $t+1$. Champ: emploi total.

Source: Enquête Histoire de vie, Insee, 2003. 
de chômage ou d'inactivité intercalée. Le taux de changement professionnel(7) mesure ces mobilités, qu'il s'agisse de changements de profession avec ou sans changement de statut et/ou de qualification. Les graphiques $3 \mathrm{a}$ et $3 \mathrm{~b}$ sur l'évolution du taux de changement professionnel par âge pour les trois générations montrent une certaine stabilité de la mobilité interne à l'emploi pour les hommes. Le fait qu'il existe un risque de sous-estimation des mobilités anciennes (souligné dans l'encadré 1) renforce plus qu'il n'affaiblit ce résultat de relative stabilité. Concernant les femmes, on observe une légère augmentation de la mobilité pour celles nées entre 1960 et 1969(8). Malgré l' «effet rattrapage» (hausse supérieure de la mobilité pour les femmes), le taux de changement professionnel reste plus élevé pour les hommes avec un écart d'environ deux points. Néanmoins, sur l'ensemble de la période, ce taux est relativement faible, puisqu'au maximum il atteint $16 \%$, ce qui signifie qu'au plus un sixième des individus de notre échantillon connaît un changement professionnel dans l'emploi entre $t$ et $t+1$.

Encore une fois, ces graphiques font apparaître un effet «cycle de vie». Quelle que soit la cohorte concernée, la mobilité atteint un pic, entre 17 et 26 ans pour les hommes, et entre 17 et 24 ans pour les femmes, puis décroît avec l'âge (9) et tombe à des taux très faibles aux alentours de 50 ans (moins de $2 \%$ ). Deux explications possibles de cette forte mobilité des jeunes qui ne s'excluent pas. Premièrement, ce serait une mobilité «positive», constituée essentiellement de changements de professions volontaires. Deuxièmement, nous rappelons que les courtes périodes de chômage ne sont pas comptabilisées comme années de chômage, elles peuvent donc être incluses dans notre indicateur du taux de changement professionnel si elles sont suivies d'un changement de profession lors du retour dans l'emploi. Or, les jeunes sont davantage touchés par le chômage que les individus d'âge médian, et par ailleurs on sait que leurs durées de chômage sont plutôt courtes. DUPRAY (2005) montre également que les jeunes se caractérisent par une mobilité externe et une mobilité interne supérieure, mais précise que celles-ci concernent des publics différents: les mobilités internes correspondent à des progressions salariales tandis que les mobilités externes sont des stratégies pour corriger les mauvaises insertions professionnelles. Finalement, il individus en emploi en $t$ avec un changement professionnel entre $t$ et $t+1$

individus en emploi en $t$

(8) Cela reste vrai même en tenant compte de l'âge retardé de l'entrée des femmes dans la vie active observé dans le graphique $3 \mathrm{~b}$.

(9) Le profil par âge des hommes et des femmes n'est cependant pas exactement le même. Pour les hommes, on observe un creux vers 20 ans (de moins en moins important), creux absent du profil par âge des femmes. Il s'agit sans doute là d'un effet des mouvements masculins de main-d'œuvre vers l'inactivité pour cause d'engagements militaires. faut être prudent quant à l'interprétation de l'augmentation du taux de changement professionnel. Il ne s'agit pas forcément d'une hausse de la mobilité volontaire et positive mais sans doute aussi d'une hausse de la précarité, notamment concernant les plus jeunes.

\section{... mais surtout à une hausse de l'insécurité, véritable nouvelle donne des carrières}

La hausse de la précarité est aussi visible à travers le taux de transition de l'emploi privé vers le chômage (taux d'insécurité(10)). Les graphiques $4 \mathrm{a}$ et $4 \mathrm{~b}$ par année (11) montrent que l'insécurité sur le marché du travail s'est accrue, pour toutes les générations, et en particulier depuis le début des années quatre-vingt. La hausse est relativement forte puisque ce taux, qui était quasiment nul dans les années soixante, atteint $1 \%$ dans les années soixante-dix $(0,5 \%$ pour les hommes), $2 \%$ dans les années quatre-vingt ( $1,5 \%$ pour les hommes) et des valeurs entre 2,5 et $5 \%$ dans les années quatre-vingt-dix ( 2 et $4 \%$ pour les hommes). Il semble donc qu'il s'agisse d'une augmentation en tendance, au-delà des fluctuations cycliques, avec une inégalité persistante concernant les risques de chômage entre hommes et femmes, mais au final, le taux d'insécurité reste relativement faible sur le marché du travail français.

Ce taux est très erratique, fonction de la conjoncture économique, mais celle-ci ne semble pas affecter exactement de la même manière et au même moment toutes les générations. Sur la période 1982-2002, pour les hommes d'âge médian [195059], on retrouve à peu près les mêmes évolutions du taux d'insécurité que celles décrites par Fougère (2003), et avec les mêmes dates charnières que lui(12): croissance de l'insécurité de 1981 à 1984 (de 0,6 à 1,3\%), puis baisse jusqu'en $1990(0,2 \%)$, forte hausse ensuite jusqu'en 1996 (2,3\%) et baisse importante (jusqu'à $0,7 \%$ ) qui s'achève par une remontée en fin de période. La génération suivante [1960-69] connaît des évolutions comparables, du moins au début de la fenêtre d'observation. À la fin, leur courbe d'insécurité «colle» mieux que celle de individus en emploi privé en $t$ et au chômage entre $t$ et $t+1$

individus en emploi privé en $t$

(11) Le graphique par année et non par âge permet de montrer la dégradation du marché du travail à partir des années soixantedix et donc l'apparition du chômage dans les carrières, ce qui est l'effet dominant. L'effet cycle de vie étant limité, les graphiques par âge sont peu lisibles. Par ailleurs, cela nous permet aussi de comparer nos résultats aux autres travaux sur l'insécurité utilisant le même indicateur et ainsi de tester la fiabilité de nos données par rapport à celles de l'enquête Emploi à partir desquelles les travaux sur l'insécurité et l'instabilité sont généralement réalisés.

(12) Mais non pas avec les mêmes niveaux d'insécurité puisque l'on ne mesure l'insécurité que vers du chômage de longue durée. 
Graphique 4a

Taux d'insécurité des hommes depuis 1970

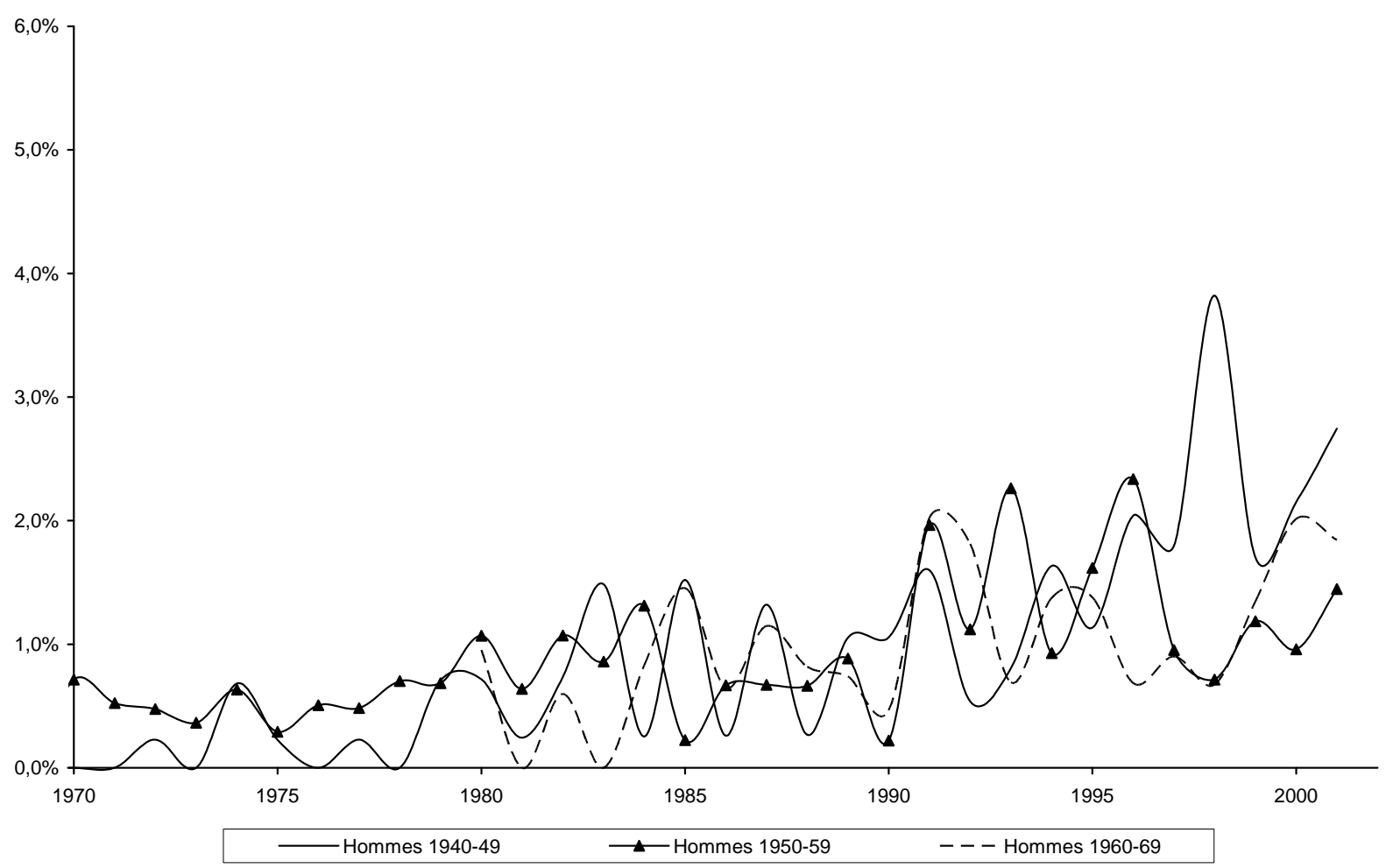

Graphique 4b

Taux d'insécurité des femmes depuis 1970

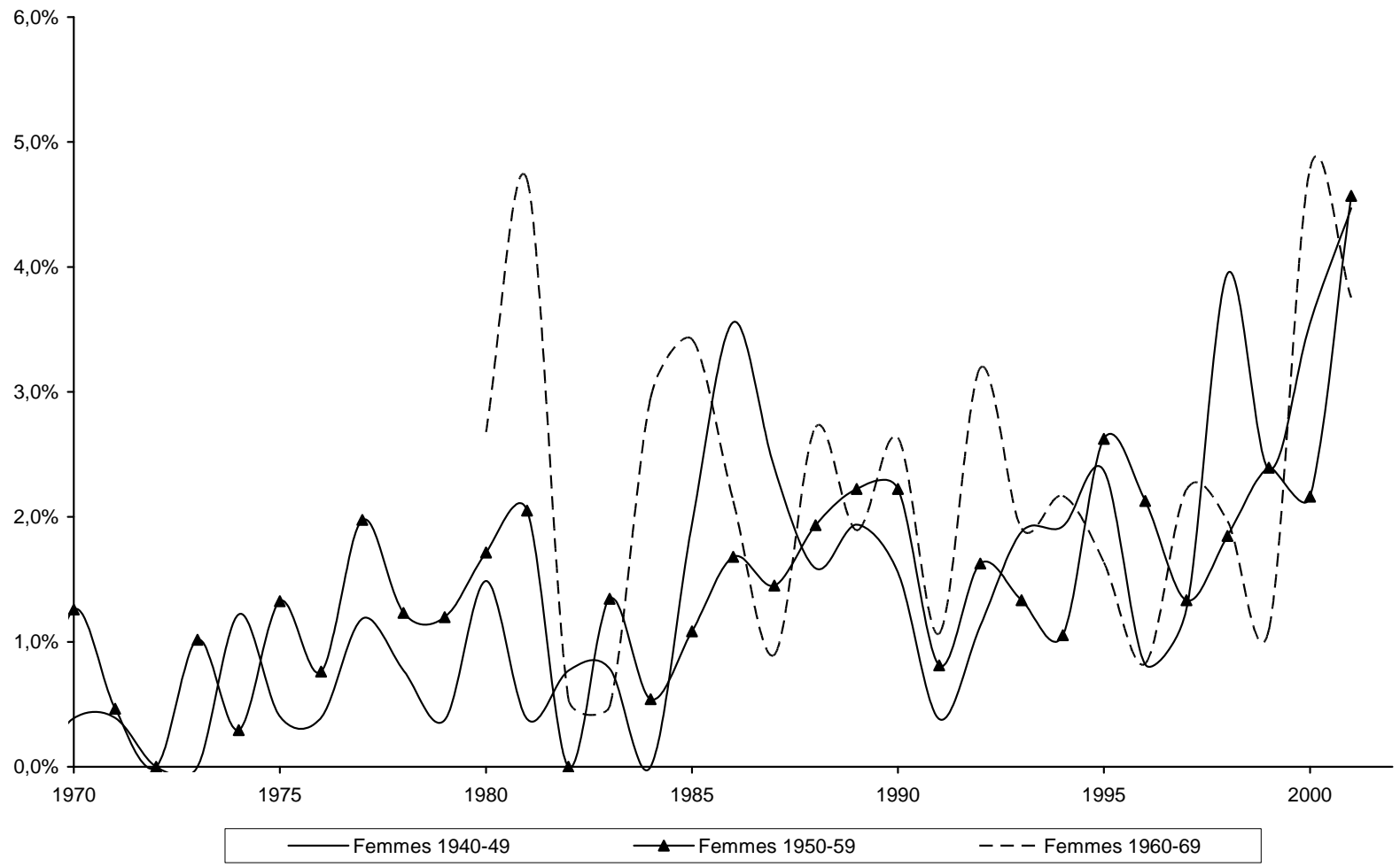

Indicateur: pourcentage des personnes d'une génération donnée, salariées dans l'emploi privé en $t$, au chômage en $t+1$.

Champ: emploi salarié privé.

Source: Enquête Histoire de vie, Insee, 2003. 
leurs aînés aux évolutions conjoncturelles. Enfin, la génération des «seniors» (la génération [1940-49]) est caractérisée par des évolutions contracycliques ou déconnectées des cycles à la fin de la fenêtre d'observation (c'est-à-dire lorsqu'elle atteint 55 ans); même en période de reprise, son taux d'insécurité continue d'augmenter et atteint même un pic de 3,8\% en 1998 alors qu'au même moment le taux d'insécurité des deux autres générations est seulement de $0,7 \%$. Il semble bien que ce soit l'âge qui soit en cause. Ces graphiques, outre l'effet dominant de dégradation générale du marché du travail, exposent donc en creux les inégalités d'âge face au chômage et illustrent la politique de gestion de la main-d'œuvre à la française : sécurité d'emploi pour la population d'âge médian (les 34-53 ans en 2003) et report des ajustements sur les âges extrêmes, les jeunes(13) et les seniors. Ils montrent également une insécurité plus forte pour les femmes que pour les hommes (écart d'environ un point).

\section{Une évolution des mobilités non uniforme}

Nous poursuivons l'analyse en cherchant à contrôler l'effet de différentes variables sur l'évolution des mobilités depuis 1960. Il s'agit de consolider notre première conclusion «toutes choses égales par ailleurs », à savoir une hausse des mobilités sur le marché du travail français d'une génération à l'autre, et de tenter de voir si cette hausse des mobilités a été uniforme ou s'est concentrée sur certaines catégories.

Pour ce faire, on définit une large population d'observations où l'unité n'est pas un individu, mais un couple d'années consécutives sur le marché du travail (emploi ou chômage, $c f$. encadré 2). On procède ainsi comme s'il s'agissait d'individus différents à chaque nouvelle transition. Des effets fixes inobservables sont donc susceptibles de biaiser l'estimation. Une partie de ce phénomène est ensuite contrôlée par l'introduction de variables résumant le type de carrière déjà connue, notamment en termes de mobilité passée. La transition est caractérisée par les attributs de l'individu en $t$, et on teste à l'aide de régressions logistiques binomiales les corrélations entre ces attributs et la probabilité que la transition entre $t$ et $t+l$ soit une mobilité, un changement professionnel, une sortie de l'emploi ou un retour dans l'emploi.

\section{Encadré 2}

\section{Les transitions sur le marché du travail}

Les observations de notre population ne sont pas des individus mais des transitions entre des états d'une année $t$ à une année $t+1$.

Nous qualifions de transition la succession de deux états, même identiques, sur le marché du travail: chômage-emploi, emploi-chômage, mais aussi chômage-chômage et emploi-emploi (avec un changement professionnel ou non). Sont sélectionnées toutes les transitions des individus nés entre 1940 et 1969 , ayant, en $t$, entre 20 ans et, au plus, 54 ans (pour la génération [1940-49]), 44 ans (pour la génération [1950-59]) et 34 ans (pour la génération [1960-69]) (1), avec déjà au moins un an d'expérience professionnelle en France et ne sortant pas vers l'étranger ou l'inactivité en $t+1$. De la sorte, nous avons écarté les années de première entrée dans l'emploi, les années de chômage des primo demandeurs, et les transitions hors du marché du travail (français), en assimilant le marché du travail à l'activité (emploi + chômage).

La population compte alors 86592 observations, soit autant de transitions ou de couples d'années $(t, t+1)$ définies au sein du marché du travail; 6021 de ces transitions présentent une mobilité. Les observations les plus anciennes remontent à 1960, l'année $t$ la plus récente étant 2002.

Le premier ensemble de régressions (cf. tableau 1) porte sur la probabilité de connaître une mobilité entre $t$ et $t+1$ (tous types de mobilité confondus); le champ est dans ce cas la population qui vient d'être définie. Puis, en fonction du type de mobilité que l'on veut analyser, on restreint le champ aux seuls couples d'années consécutivement dans l'emploi (pour la probabilité d'un changement professionnel), ou tels que l'année $t$ est en emploi (pour la probabilité de sortie vers le chômage), ou encore tels que l'année $t$ est une année de chômage (pour la probabilité de retour dans l'emploi) (cf. tableaux 2 et 3 ).

(1) Les bornes d'âge supérieures ont été choisies telles que tous les individus d'une génération donnée aient atteint l'âge maximum considéré.

(13) Nous n'avons pas dans cet article inclus la génération la plus récente de l'enquête [1970-79] pour limiter les biais de sélection (seuls les moins diplômés étaient entrés sur le marché du travail au moment de l'enquête en 2003) mais on constate pour eux des évolutions du taux d'insécurité très fortement liées à l'état du marché du travail : très forte baisse du taux d'insécurité à la fin des années quatre-vingt-dix et remontée importante depuis 2000 . 


\section{L'ensemble des mobilités}

Dans les régressions (1) à (3) du tableau 1, on estime l'évolution des mobilités (tous types confondus) de génération en génération, en cherchant à contrôler d'éventuels effets de structure. L'analyse est faite ici, hommes et femmes regroupés, pour analyser l'évolution agrégée des mobilités dans les carrières. Dans les régressions suivantes ( $c f$. tableaux 2 et 3 ) qui détaillent chaque type de mobilité (changements professionnels, sorties vers le chômage et retours en emploi), nous séparons les hommes et les femmes afin de vérifier si les évolutions sont semblables pour ces deux populations.

La variable principale croise générations et classes d'âge. La période de cycle de vie entre 30 et 34 ans de la génération [1950-59] est prise comme modalité de référence. Nous testons successivement trois modèles en introduisant chaque fois de nouvelles variables. Le premier modèle $(c f$. tableau $1,(1))$ qui teste l'augmentation de la mobilité totale (en régressant le Logit de la probabilité d'une mobilité entre $t$ et $t+1)$, contrôlée partiellement des variations conjoncturelles (14), confirme la tendance à la hausse observée graphiquement dans la partie précédente. À classe d'âge donnée, la probabilité d'une mobilité augmente génération après génération et particulièrement pour la dernière [1960-69]. À génération donnée, la probabilité d'une mobilité décroît avec l'âge; en particulier, la classe d'âge des 20-24 ans et, de façon moindre, les 25-29 ans et 30-34 ans, quelle que soit la génération, se distinguent des plus de 35 ans par leur taux de mobilité très supérieur. On retrouve ainsi les effets bruts de "génération» et de «cycle de vie» que nous avions observés dans la première partie. Cela rejoint les résultats d'autres travaux, notamment ceux de CHAPOULIE (2000), qui, à partir de l'enquête FQP observe une montée spectaculaire de la mobilité chez les 20-24 ans et une distinction nette entre les moins de 40 ans (qui cumulent plus grande vulnérabilité au chômage et changements professionnels plus fréquents) et les plus de 40 ans (qui connaissent des carrières plus stables). Pour questionner ces premiers effets, on introduit dans le modèle suivant ( $c f$. tableau $1,(2))$, un ensemble de variables venant contrôler les possibles effets de composition liés aux transformations de la population active, en particulier ceux liés à la féminisation de la main-d'œuvre et à l'augmentation du niveau de formation.

On constate que les femmes ont une probabilité moindre que les hommes d'être mobiles, alors qu'il n'y a pas d'effet significatif pour les immigrés (15). Par ailleurs, les salariés du public et les indépendants

(14) En introduisant la moyenne annuelle du taux de variation du PIB par demi-décennie.

(15) Nous conservons néanmoins cette variable dans la régression car elle permet de contrôler la surreprésentation dans l'enquête des personnes d'origine étrangère. sont moins mobiles que les salariés du privé. En ce qui concerne la formation initiale, plus le niveau d'études est élevé, plus la mobilité est forte toutes choses égales par ailleurs, et en particulier à catégorie socioprofessionnelle égale. De fait, l'effet du niveau de catégorie socioprofessionnelle joue dans le sens contraire de celui du diplôme (par rapport aux professions intermédiaires, les plus mobiles sont les ouvriers non qualifiés, les employés qualifiés et les employés non qualifiés (16), les moins mobiles les cadres et les ouvriers qualifiés). Première interprétation possible de ce résultat: pour les catégories socioprofessionnelles «basses», les chances de connaître une mobilité, notamment d'être promu, sont d'autant plus élevées que le niveau de diplôme est élevé; concernant les catégories socioprofessionnelles "hautes», elles peuvent correspondre pour les uns au sommet d'une carrière ascendante (les moins diplômés) et pour les autres à une simple étape (les plus diplômés). Par exemple, un cadre a d'autant plus de chances de bouger qu'il est devenu cadre jeune (c'est-à-dire qu'il a un diplôme élevé)(17), alors qu'un ouvrier ou un employé qualifié qui passe cadre par promotion interne arrive au bout de sa chaîne de mobilité et sera relativement immobile par la suite (il n'a plus guère de possibilité de promotion interne et aura des difficultés à faire reconnaître son évolution acquise en interne s'il venait à changer d'entreprise ou de profession, ce qui limite aussi sa mobilité externe). Seconde explication possible, on aurait ici la trace de deux types de mobilités: une positive, faite de promotions, plus probable pour les hauts niveaux de diplôme, et une plus négative, correspondant à une forte instabilité et insécurité pour les emplois de premier niveau de qualification. Amossé (2003) montre bien cette dualisation de la mobilité (externe), de plus en plus souvent associée à des promotions mais également de plus en plus souvent synonyme de flexibilité.

Ainsi, les transformations de la population active ont sans doute conduit, pour une part, à l'augmentation de la mobilité sur le marché du travail (notamment l'augmentation du niveau d'études et la baisse de la part des indépendants dans la population active) et, pour une autre, à sa baisse (entrée massive des femmes dans la population active, accroissement du nombre de cadres). A priori, on ne peut donc pas prédire l'évolution globale de la mobilité. En fait, ces effets de composition étant contrôlés, on constate toujours une hausse de la

(16) Pour la distinction entre employés qualifiés et non qualifiés nous avons utilisé une approximation de la méthode de Burnod et Chenu (2001), issue du croisement entre la catégorie socioprofessionnelle à deux chiffres et le statut public ou privé de l'emploi. Nous opposons les employés non qualifiés, d'une part, aux employés qualifiés et employés de la Fonction publique, d'autre part.

(17) Plusieurs travaux montrent l'émergence d'un marché externe relativement plus ouvert et plus mobile pour les plus diplômés. $C f$. GERME (2001). 
mobilité mesurée par la variable génération $\mathrm{x}$ âge mais quelque peu atténuée par rapport au modèle (1). Il ressort encore plus nettement que les générations [1940-49] et [1950-59] se distinguent peu en termes de mobilité et que par contre, une vraie hausse de la mobilité s'est produite pour la dernière génération [1960-69] (avec toujours un risque de sous-estimation des mobilités les plus anciennes). L'effet cycle de vie reste très marqué. L'effet positif de la conjoncture est plus marqué et plus significatif que dans le premier modèle; il laisse penser qu'en période de récession, le recul des mobilités volontaires par peur du chômage fait plus que compenser la hausse des mobilités contraintes due à la mauvaise conjoncture.

Le modèle (3) introduit deux variables pour contrôler le type de la carrière déjà connue quand survient la mobilité (et donc contrôle le rang de la mobilité considérée): le nombre de changements de catégorie socioprofessionnelle depuis le premier emploi(18) et le fait d'avoir ou non connu du chômage dans le passé(19). On constate que le chômage et la mobilité socioprofessionnelle passés sont positivement corrélés à la probabilité de connaître une mobilité, conformément au résultat bien connu selon lequel la mobilité entraîne la mobilité (Granovetter, 2000). Amossé (2003) montre d'ailleurs que la moitié des personnes qui changent d'employeur dans l'année ont moins de deux ans d'ancienneté. Le reste du modèle est très stable, les effets commentés précédemment sont donc robustes.

\section{Les mobilités au sein de l'emploi et avec le chômage}

À présent nous décomposons les mobilités en trois mouvements distincts déjà définis dans la première partie: les changements professionnels, les sorties de l'emploi et les retours dans l'emploi. Pour les deux premiers types de mouvements, le nombre important d'observations nous permet également de distinguer hommes et femmes. On procède pour chaque mobilité en deux temps: on teste d'abord ces modèles sans intégrer les variables contrôlant la mobilité passée ( $c f$. tableau 2) puis on ajoute les variables «nombre de changements de catégorie socioprofessionnelle» et "chômage connu dans le passé» ( $c f$. tableau 3$)$.

Si l'on s'intéresse d'abord aux variables contrôlant la composition de la population active, on constate cette fois, mais pour les hommes seule-

(18) Étant donné la construction de nos variables, tout changement de catégorie socioprofessionnelle implique une mobilité, mais la mobilité dont on estime la probabilité d'occurrence n'implique pas forcément un changement de catégorie socioprofessionnelle.

(19) Pour les chômeurs à la date $t$, on ne compte pas l'épisode présent de chômage. ment, que le fait d'être immigré est corrélé négativement avec la probabilité de connaître un changement professionnel, alors que cette variable n'est pas significativement liée avec le risque d'entrée et les chances de sortie du chômage. Comme pour les mobilités totales, le diplôme est corrélé positivement avec les changements professionnels alors que l'effet du niveau de catégorie socioprofessionnelle va dans le sens contraire. Par contre, il n'y a pas de lien significatif entre diplôme et insécurité : c'est le fait d'appartenir à une catégorie socioprofessionnelle «basse» (employés et ouvriers non qualifiés pour les hommes, ouvrières pour les femmes) qui pèse sur le risque de chômage, c'està-dire le fait d'occuper des emplois instables; et le fait d'avoir un faible niveau de diplôme est plutôt associé à une faible probabilité d'un retour en emploi lorsque l'on est au chômage. Ce poids très important du diplôme dans les recrutements en France a fait l'objet de nombreux travaux, notamment MARChal et Torny (2003). Les fortes inégalités face au chômage touchant les non qualifiés seraient donc dues à deux éléments : une vulnérabilité plus forte des emplois de premier niveau de qualification et une employabilité jugée plus faible des peu diplômés, du fait des critères de sélection en vigueur sur le marché du travail français. Enfin, le statut antérieur de l'emploi public ou hors salariat protège du risque de chômage et est corrélé à une plus faible mobilité interne à l'emploi. Par contre, avoir occupé un emploi public avant d'entrer au chômage (ce qui révèle a posteriori l'occupation d'un emploi précaire du service public) est associé à un risque légèrement supérieur (avec une significativité à $10 \%$ ) de rester chômeur.

Observons à présent l'impact de la variable génération $\mathrm{x}$ âge. Concernant les changements professionnels ( $c f$. tableau 2,(1)), l'effet cycle de vie (mobilité décroissante avec l'âge) est toujours présent et même un peu plus marqué. En revanche, on constate cette fois une certaine stabilité des mobilités internes à l'emploi voire une diminution pour les hommes : par exemple, les 25-29 ans de la génération [1940-49] sont plus mobiles que ceux des générations [195059] et [1960-69], alors même que c'est plutôt une sous-estimation de la mobilité des [1940-49] que l'on craint. Cela pourrait s'expliquer par une baisse des promotions (Goux, 1991). C'est seulement chez les femmes que l'on constate une augmentation des changements professionnels, et uniquement pour la dernière génération. La conjoncture affecte positivement la mobilité féminine au sein de l'emploi alors qu'elle n'a pas d'effet significatif pour les hommes. Les femmes, qui, malgré cette plus forte hausse des mobilités, connaissent en moyenne moins de changements professionnels $(4,7 \%$ contre $6,2 \%$ ), doivent attendre, plus que les hommes, une amélioration de la conjoncture pour connaître une mobilité au sein de l'emploi. On pressent là le rôle de variable d'ajustement que l'on a longtemps fait 
Tableau 1

Régressions du Logit de la probabilité d'une mobilité sur le marché du travail (1960-2002)

\begin{tabular}{|c|c|c|c|c|c|c|}
\hline & \multicolumn{6}{|c|}{ Mobilité sur le marché du travail entre $t$ et $t+1$} \\
\hline & \multicolumn{2}{|c|}{ (1) } & \multicolumn{2}{|c|}{$(2)$} & \multicolumn{2}{|c|}{$(3)$} \\
\hline Probabilité moyenne de l'événement: & \multicolumn{2}{|c|}{$7,0 \%$} & \multicolumn{2}{|c|}{$7,0 \%$} & \multicolumn{2}{|c|}{$7,0 \%$} \\
\hline & Coefficient & Écart type & Coefficient & Écart type & Coefficient & Écart type \\
\hline Constante & $-2,84 * * *$ & 0,07 & $-2,74 * * *$ & 0,08 & $-2,86 * * *$ & 0,08 \\
\hline \multicolumn{7}{|l|}{$\begin{array}{l}\text { Génération x classe d'âge } \\
\text { (réf.: [50-59] } \times 30-34 \text { ans): }\end{array}$} \\
\hline [1940-49] x 20-24 ans & $0,51 * * *$ & 0,08 & $0,48 * * *$ & 0,09 & $0,55 * * *$ & 0,09 \\
\hline$[1940-49] \times 25-29$ ans & $0,24 * *$ & 0,08 & $0,24 * *$ & 0,08 & $0,29 * * *$ & 0,08 \\
\hline$[1940-49] \times 30-34$ ans & 0,01 & 0,08 & 0,06 & 0,08 & 0,07 & 0,08 \\
\hline [1940-49] x 35-39 ans & $-0,25 * *$ & 0,08 & $-0,17 *$ & 0,08 & $-0,19 *$ & 0,08 \\
\hline [1940-49] x 40-44 ans & $-0,27 * * *$ & 0,08 & $-0,18 *$ & 0,08 & $-0,22 * *$ & 0,08 \\
\hline [1940-49] x 45-49 ans & $-0,32 * * *$ & 0,08 & $-0,22 * *$ & 0,08 & $-0,29 * * *$ & 0,08 \\
\hline [1940-49] x 50-54 ans & $-0,36 * * *$ & 0,09 & $-0,25 * *$ & 0,09 & $-0,35 * * *$ & 0,09 \\
\hline$[1950-59] \times 20-24$ ans & $0,59 * * *$ & 0,07 & $0,52 * * *$ & 0,07 & $0,58 * * *$ & 0,07 \\
\hline$[1950-59] \times 25-29$ ans & $0,23 * *$ & 0,07 & $0,19 * *$ & 0,07 & $0,23 * *$ & 0,07 \\
\hline$[1950-59] \times 35-39$ ans & 0,00 & 0,07 & 0,02 & 0,07 & $-0,02$ & 0,07 \\
\hline$[1950-59] \times 40-44$ ans & $-0,06$ & 0,07 & $-0,02$ & 0,07 & $-0,09$ & 0,07 \\
\hline$[1960-69] \times 20-24$ ans & $0,86 * * *$ & 0,07 & $0,73 * * *$ & 0,07 & $0,78 * * *$ & 0,07 \\
\hline$[1960-69] \times 25-29$ ans & $0,52 * * *$ & 0,07 & $0,42 * * *$ & 0,07 & $0,43 * * *$ & 0,07 \\
\hline [1960-69] x 30-34 ans & $0,27 * * *$ & 0,07 & $0,20 * *$ & 0,07 & $0,16 *$ & 0,07 \\
\hline Femme (réf.: Homme) & & & $-0,32 * * *$ & 0,03 & $-0,30 * * *$ & 0,03 \\
\hline Immigré (réf.: Non immigré) & & & $-0,07$ & 0,04 & $-0,03$ & 0,04 \\
\hline $\begin{array}{l}\text { Niveau d'études atteint (réf.: Enseignement } \\
\text { technique ou professionnel court): }\end{array}$ & & & & & & \\
\hline Aucune étude à dernière année d'études & & & & & & \\
\hline primaires & & & $-0,18 * * *$ & 0,04 & $-0,18 * * *$ & 0,04 \\
\hline Premier cycle d'enseignement général & & & $-0,09$ & 0,05 & $-0,09$ & 0,05 \\
\hline Deuxième cycle d'enseignement général & & & $0,24 * * *$ & 0,05 & $0,27 * * *$ & 0,05 \\
\hline Enseignement technique ou professionnel & & & & & & \\
\hline long & & & $0,24 * * *$ & 0,06 & $0,26 * * *$ & 0,06 \\
\hline Enseignement supérieur & & & $0,46 * * *$ & 0,05 & $0,49 * * *$ & 0,05 \\
\hline \multicolumn{7}{|l|}{$\begin{array}{l}\text { Catégorie socioprofessionnelle en } t \text { (dernière } \\
\text { catégorie socioprofessionnelle pour les } \\
\text { chômeurs; réf.: Professions intermédiaires): }\end{array}$} \\
\hline $\begin{array}{l}\text { Agriculteurs, artisans, commerçants et } \\
\text { chefs d'entreprise }\end{array}$ & & & 0,14 & 0,11 & 0,13 & 0,11 \\
\hline Cadres & & & $-0,29 * * *$ & 0,06 & $-0,27 * * *$ & 0,06 \\
\hline Employés non qualifiés & & & $0,40 * * *$ & 0,06 & $0,38 * * *$ & 0,06 \\
\hline $\begin{array}{l}\text { Employés qualifiés et de la Fonction } \\
\text { publique }\end{array}$ & & & $0,18 * * *$ & 0,04 & $0,17 * * *$ & 0,05 \\
\hline Ouvriers non qualifiés & & & $0,47 * * *$ & 0,05 & $0,48 * * *$ & 0,05 \\
\hline Ouvriers qualifiés & & & $-0,08$ & 0,05 & $-0,09$ & 0,05 \\
\hline \multicolumn{7}{|l|}{$\begin{array}{l}\text { Statut de l'emploi en } t \text { (dernier emploi pour les } \\
\text { chômeurs; réf. : Emploi salarié dans le privé): }\end{array}$} \\
\hline Emploi salarié dans le public & & & $-0,47 * * *$ & 0,04 & $-0,46 * * *$ & 0,04 \\
\hline Emploi hors du salariat & & & $-0,58 * * *$ & 0,10 & $-0,58 * * *$ & 0,10 \\
\hline \multicolumn{7}{|l|}{$\begin{array}{l}\text { Nombre de changements de catégorie socio- } \\
\text { professionnelle (à } 2 \text { chiffres) depuis le premier } \\
\text { emploi (réf.: aucun): }\end{array}$} \\
\hline Un & & & & & $-0,01$ & 0,03 \\
\hline Deux & & & & & $0,12 * *$ & 0,04 \\
\hline Trois et plus & & & & & $0,52 * * *$ & 0,05 \\
\hline $\begin{array}{l}\text { A connu du chômage dans le passé (réf.: N'a } \\
\text { jamais connu de période de chômage) }\end{array}$ & & & & & $0,34 * * *$ & 0,05 \\
\hline $\begin{array}{l}\text { Moyenne annuelle du taux de variation du PIB } \\
\text { par demi-décennie (variable continue) }\end{array}$ & 0,03 & 0,02 & $0,03 *$ & 0,02 & $0,04 *$ & 0,02 \\
\hline $\begin{array}{l}\text { Nombre d'observations } \\
\text { Pourcentage de paires concordantes }\end{array}$ & \multicolumn{2}{|c|}{86592} & \multicolumn{2}{|c|}{86592} & \multicolumn{2}{|c|}{$63,8 \%$} \\
\hline
\end{tabular}

Champ: population active ayant déjà travaillé.

$* * *$ : significatif à $0,1 \% ; * *$ : significatif à $1 \% ; *$ : significatif à $5 \%$; coefficient en italique: significatif à $10 \%$.

réf.: modalité de référence.

Source: Enquête Histoire de vie, Insee, 2003.

- 24 - Travail et Emploi $n^{\circ} 113$ • Janvier-mars 2008 
endosser aux femmes sur le marché du travail, et encore aujourd'hui dans une certaine mesure. Cette moindre mobilité «interne» à l'emploi des femmes n'est cependant pas forcément que négative. LECLAIR et Roux (2005) montrent que, toutes choses égales par ailleurs, les femmes occupent des emplois moins instables que les hommes.

En ce qui concerne les sorties de l'emploi ( $c f$. tableau 2, (2)) une bonne conjoncture protège significativement du risque de chômage les hommes mais non les femmes. Leur risque moyen respectif est très faible sur la période étudiée: $0,7 \%$ pour les uns et $1,1 \%$ pour les autres. À conjoncture donnée, l'effet de la variable "génération $\mathrm{x}$ âge » est le suivant: comparativement à la population de référence, les hommes et les femmes de la génération [1940-49] subissent moins le risque de mise au chômage entre 20 et 39 ans, mais beaucoup plus après 50 ans. Cette génération [1940-49], qui est entrée sur le marché du travail dans les années soixante et qui en 2003 était âgée de 54 à 63 ans illustre parfaitement la montée persistante, décennie après décennie, du risque de chômage dans les carrières. Il s'agit donc là d'un effet date (montée forte du risque de chômage à partir de la fin des années soixante-dix). Par ailleurs, on observe un risque de chômage particulièrement fort pour les hommes de la génération [1940-49] âgés de plus de 50 ans (dans les années quatre-vingt-dix) et, parmi les femmes, pour les jeunes de la génération [1960-69] (dès les années quatre-vingt). Nous avons là deux manifestations de la gestion des âges à la française déjà évoquée: dans les années récentes, sont sortis plus fréquemment de l'emploi pour le chômage les jeunes et les seniors.

Enfin, c'est clairement un effet âge ou «fin de carrière» qui ressort de la dernière régression sur les sorties du chômage. Les coefficients significatifs concernent des individus âgés de 40 à 55 ans. Leur chance de sortie du chômage est fortement diminuée par rapport à la population de référence. On sait que l'âge est aujourd'hui un obstacle très important au recrutement, obstacle qui a même été institutionnalisé puisque depuis 1984 les chômeurs peuvent, sous certaines conditions, être affranchis des démarches de recherche d'emploi(20). Enfin, le taux de variation du PIB contrôle de manière positive, mais peu significative la probabilité de retour dans l'emploi ( $c f$. tableau 2, (5)), probabilité dans notre échantillon qui s'élève en moyenne à $23,7 \%$. Dans cette dernière régression, le nombre d'observations ne nous a pas permis de séparer hommes et femmes, mais on constate que la variable genre

(20) La dispense de recherche d'emploi (DRE), sous sa forme actuelle, permet à un demandeur d'emploi âgé de 55 ans ou plus d'être dispensé de cette recherche, sous conditions de durée de cotisation, après en avoir fait volontairement la demande auprès de son agence locale pour l'emploi. À partir de 57 ans et demi, tous les allocataires du régime d'assurance chômage sont potentiellement concernés. contrôle un effet fort et très significatif au détriment des femmes.

Concernant les variables supplémentaires de carrières ajoutées dans le tableau 3 , on constate que la mobilité passée est corrélée positivement à la probabilité de connaître un changement professionnel, ainsi qu'au risque de chômage tout en étant positivement corrélée aux chances d'en sortir. De son côté, le chômage passé est associé à un risque supérieur de mise au chômage mais n'a pas d'impact significatif sur les chances d'en sortir. Ces résultats mènent à un diagnostic simple: toutes choses égales par ailleurs, la stabilité entraîne la stabilité, l'instabilité nourrit l'instabilité, le chômage crée du chômage. Bien sûr, rien ici ne nous permet de savoir si c'est l'instabilité (le chômage) passée qui détermine l'instabilité (le chômage) future (à partir d'individus initialement identiques) ou si certains individus instables créent des parcours instables (qui n'ont pas de raison de se stabiliser après un certain nombre de positions professionnelles). C'est le problème classique de l'hétérogénéité ex post ou ex ante (HECKMAN, BORJAS, 1980). Il n'en demeure pas moins que les mobilités semblent se concentrer sur certaines carrières, alors que dans notre échantillon, en 2003 , plus de $60 \%$ des individus de chaque génération n'ont connu dans leur carrière qu'une seule ou deux catégories socioprofessionnelles (21). Cette mobilité est à la fois favorable et défavorable au sens où elle accroît la vulnérabilité au chômage (de longue durée) mais est également source d'une plus forte employabilité et donc de plus grandes chances de sortie du chômage.
(21) Cf. annexe. 
Tableau 2.1

Régressions du Logit de la probabilité d'un changement professionnel au sein de l'emploi (1960-2002)

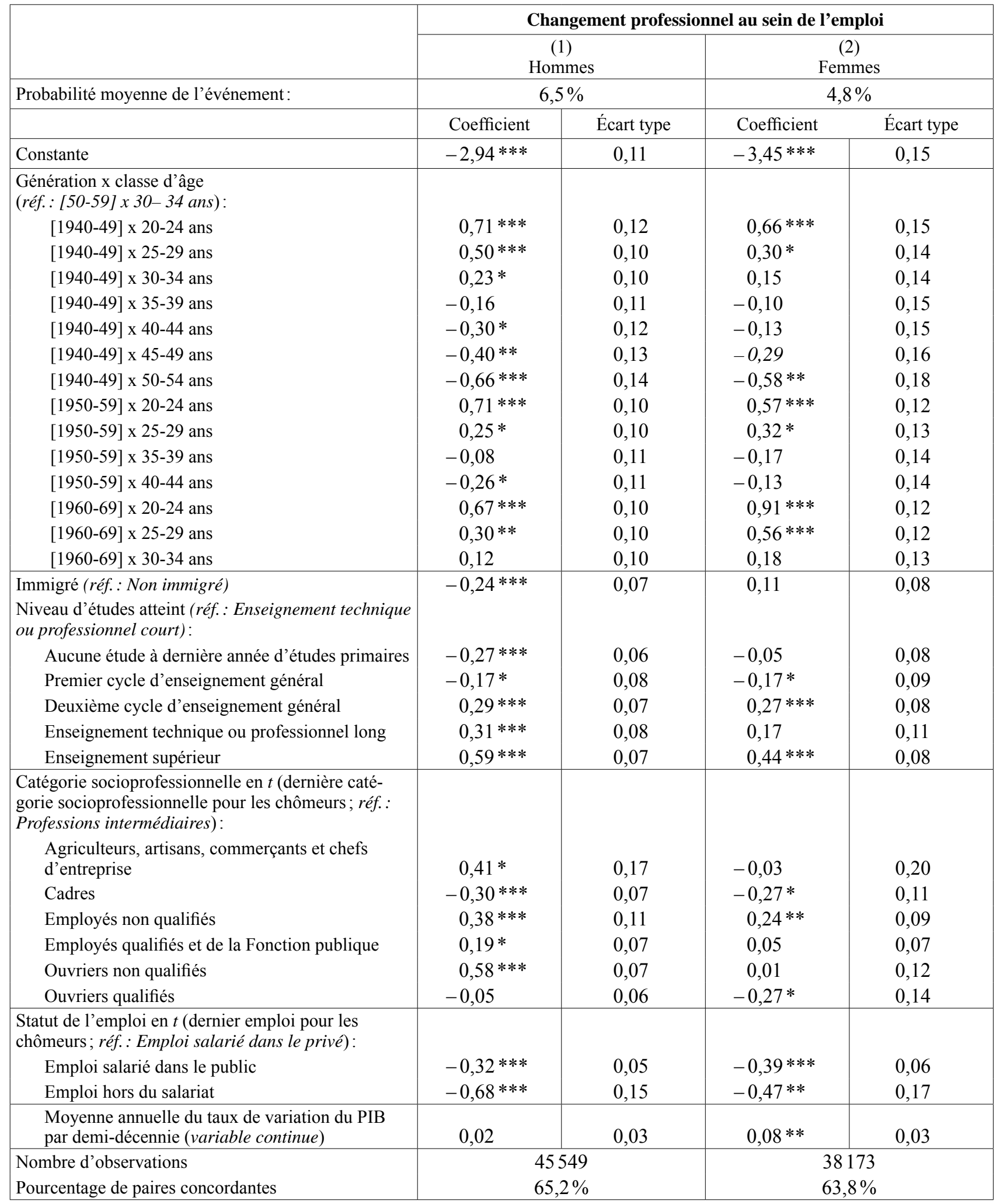

$* * *$ : significatif à $\mathbf{0 , 1} \% ; * *$ : significatif à $1 \% ; *$ : significatif à $5 \%$; coefficient en italique : significatif à $10 \%$.

réf.: modalité de référence.

Source: Enquête Histoire de vie, Insee, 2003. 
Tableau 2.2

Régressions du Logit de la probabilité d'une sortie de l'emploi vers le chômage et du retour dans l'emploi (1960-2002)

\begin{tabular}{|c|c|c|c|c|c|c|}
\hline & \multicolumn{4}{|c|}{ Sortie de l'emploi vers le chômage } & \multirow{2}{*}{\multicolumn{2}{|c|}{$\frac{\text { Retour dans l'emploi }}{(5)}$}} \\
\hline & \multicolumn{2}{|c|}{$\begin{array}{c}(3) \\
\text { Hommes }\end{array}$} & \multicolumn{2}{|c|}{$\begin{array}{c}(4) \\
\text { Femmes }\end{array}$} & & \\
\hline Probabilité moyenne de l'événement: & \multicolumn{2}{|c|}{$0,7 \%$} & \multicolumn{2}{|c|}{$1,1 \%$} & \multicolumn{2}{|c|}{$23,7 \%$} \\
\hline & Coefficient & Écart type & Coefficient & Écart type & Coefficient & Écart type \\
\hline Constante & $-4,91 * * *$ & 0,32 & $-4,59 * * *$ & 0,30 & $-0,31$ & 0,33 \\
\hline \multicolumn{7}{|l|}{$\begin{array}{l}\text { Génération x classe d'âge } \\
\text { (réf.: [50-59] x 30-34 ans): }\end{array}$} \\
\hline [1940-49] x 20-24 ans & $-13,41$ & 240 & $-1,89 * *$ & 0,63 & 0,60 & 0,75 \\
\hline [1940-49] x 25-29 ans & $-1,72 * *$ & 0,63 & $-1,28 *$ & 0,50 & 0,34 & 0,82 \\
\hline [1940-49] x 30-34 ans & $-0,74$ & 0,40 & $-0,75$ & 0,39 & 0,28 & 0,47 \\
\hline$[1940-49] \times 35-39$ ans & $-0,55$ & 0,37 & $-0,33$ & 0,33 & 0,48 & 0,34 \\
\hline [1940-49] x 40-44 ans & 0,08 & 0,32 & 0,02 & 0,30 & 0,16 & 0,30 \\
\hline [1940-49] x 45-49 ans & 0,36 & 0,30 & 0,42 & 0,27 & $-0,71 *$ & 0,29 \\
\hline [1940-49] x 50-54 ans & $1,17 * * *$ & 0,26 & 0,47 & 0,28 & $-1,16 * * *$ & 0,28 \\
\hline [1950-59] x 20-24 ans & $-0,25$ & 0,34 & 0,02 & 0,28 & 0,28 & 0,31 \\
\hline$[1950-59] \times 25-29$ ans & $-0,38$ & 0,33 & $-0,02$ & 0,28 & 0,14 & 0,28 \\
\hline$[1950-59] \times 35-39$ ans & 0,37 & 0,28 & 0,45 & 0,25 & 0,04 & 0,25 \\
\hline$[1950-59] \times 40-44$ ans & $0,73 * *$ & 0,27 & $0,62 *$ & 0,24 & $-0,57 *$ & 0,25 \\
\hline [1960-69] x 20-24 ans & 0,16 & 0,31 & $0,94 * * *$ & 0,24 & 0,32 & 0,27 \\
\hline [1960-69] x 25-29 ans & 0,39 & 0,28 & $0,66 * *$ & 0,25 & 0,11 & 0,26 \\
\hline [1960-69] x 30-34 ans & 0,47 & 0,28 & 0,50 & 0,26 & $-0,42$ & 0,25 \\
\hline $\begin{array}{l}\text { Femme (réf.: Homme) } \\
\text { Immigré (réf.: Non immigré) } \\
\text { Niveau d'études atteint (réf.: Enseignement } \\
\text { technique ou professionnel court): }\end{array}$ & 0,14 & 0,16 & 0,07 & 0,15 & $\begin{array}{c}-0,70 * * * \\
0,16\end{array}$ & $\begin{array}{l}0,13 \\
0,16\end{array}$ \\
\hline $\begin{array}{l}\text { Aucune étude à dernière année d'études } \\
\text { primaires }\end{array}$ & $-0,13$ & 0,18 & $-0,04$ & 0,16 & $-0,39 *$ & 0,17 \\
\hline Premier cycle d'enseignement général & 0,17 & 0,20 & 0,19 & 0,15 & $-0,58 * * *$ & 0,16 \\
\hline Deuxième cycle d'enseignement général & 0,00 & 0,22 & 0,03 & 0,17 & $-0,31$ & 0,20 \\
\hline $\begin{array}{l}\text { Enseignement technique ou professionnel } \\
\text { long }\end{array}$ & 0,31 & 0,23 & $-0,08$ & 0,23 & $-0,02$ & 0,27 \\
\hline Enseignement supérieur & 0,36 & 0,21 & $-0,25$ & 0,18 & 0,14 & 0,20 \\
\hline \multicolumn{7}{|l|}{$\begin{array}{l}\text { Catégorie socioprofessionnelle en } t \text { (dernière } \\
\text { catégorie socioprofessionnelle pour les } \\
\text { chômeurs; réf.: Professions intermédiaires): }\end{array}$} \\
\hline $\begin{array}{l}\text { Agriculteurs, artisans, commerçants et } \\
\text { chefs d'entreprise }\end{array}$ & 0,20 & 0,51 & $-1,11 *$ & 0,45 & 0,17 & 0,51 \\
\hline Cadres & $-0,17$ & 0,23 & $-0,20$ & 0,27 & 0,17 & 0,29 \\
\hline Employés non qualifiés & $1,08 * * *$ & 0,27 & 0,10 & 0,19 & $-0,27$ & 0,21 \\
\hline $\begin{array}{l}\text { Employés qualifiés et de la Fonction } \\
\text { publique }\end{array}$ & 0,45 & 0,27 & 0,11 & 0,16 & 0,11 & 0,19 \\
\hline Ouvriers non qualifiés & $1,19 * * *$ & 0,20 & $0,42 *$ & 0,21 & $-0,83 * * *$ & 0,20 \\
\hline Ouvriers qualifiés & 0,19 & 0,19 & $0,46 *$ & 0,22 & $-0,30$ & 0,21 \\
\hline \multicolumn{7}{|l|}{$\begin{array}{l}\text { Statut de l'emploi en } t \text { (dernier emploi pour les } \\
\text { chômeurs; réf.: Emploi salarié dans le privé): }\end{array}$} \\
\hline Emploi salarié dans le public & $-1,21 * * *$ & 0,22 & $-1,08 * * *$ & 0,16 & $-0,34$ & 0,18 \\
\hline Emploi hors du salariat & $-0,80$ & 0,45 & $-0,09$ & 0,33 & $-0,46$ & 0,44 \\
\hline $\begin{array}{l}\text { Moyenne annuelle du taux de variation du } \\
\text { PIB par demi-décennie (variable continue) }\end{array}$ & $-0,16^{*}$ & 0,08 & 0,01 & 0,07 & 0,13 & 0,07 \\
\hline $\begin{array}{l}\text { Nombre d'observations } \\
\text { Pourcentage de paires concordantes }\end{array}$ & \multicolumn{2}{|c|}{45870} & \multicolumn{2}{|c|}{$\begin{array}{l}38594 \\
67,8 \%\end{array}$} & \multicolumn{2}{|c|}{$\begin{array}{l}2125 \\
68,6 \%\end{array}$} \\
\hline
\end{tabular}

$* * *$ : significatif à $0,1 \% ; * *$ : significatif à $1 \% ; *$ : significatif à $5 \%$; coefficient en italique: significatif à $10 \%$.

réf:: modalité de référence.

Source: Enquête Histoire de vie, Insee, 2003. 
Tableau 3

Effets des variables supplémentaires dans les régressions du Logit de la probabilité d'un changement professionnel au sein de l'emploi, d'une sortie de l'emploi vers le chômage et du retour dans l'emploi (1960-2002)

\begin{tabular}{|c|c|c|c|c|c|c|c|c|c|c|}
\hline & \multicolumn{4}{|c|}{$\begin{array}{l}\text { Changement professionnel } \\
\text { au sein de l'emploi }\end{array}$} & \multicolumn{4}{|c|}{ Sortie de l'emploi vers le chômage } & \multirow{2}{*}{\multicolumn{2}{|c|}{$\begin{array}{c}\begin{array}{c}\text { Retour dans } \\
\text { l'emploi }\end{array} \\
(5)\end{array}$}} \\
\hline & \multicolumn{2}{|c|}{$\begin{array}{c}(1) \\
\text { Hommes }\end{array}$} & \multicolumn{2}{|c|}{$\begin{array}{c}(2) \\
\text { Femmes }\end{array}$} & \multicolumn{2}{|c|}{$\begin{array}{c}(3) \\
\text { Hommes }\end{array}$} & \multicolumn{2}{|c|}{$\begin{array}{c}(4) \\
\text { Femmes }\end{array}$} & & \\
\hline $\begin{array}{l}\text { Probabilité moyenne de } \\
\text { l'événement: }\end{array}$ & \multicolumn{2}{|c|}{$6,5 \%$} & \multicolumn{2}{|c|}{$4,8 \%$} & \multicolumn{2}{|c|}{$0,7 \%$} & \multicolumn{2}{|c|}{$1,1 \%$} & \multicolumn{2}{|c|}{$23,7 \%$} \\
\hline & Coefficient & $\begin{array}{l}\text { Écart } \\
\text { type }\end{array}$ & Coefficient & $\begin{array}{l}\text { Écart } \\
\text { type }\end{array}$ & Coefficient & $\begin{array}{l}\text { Écart } \\
\text { type }\end{array}$ & Coefficient & $\begin{array}{l}\text { Écart } \\
\text { type }\end{array}$ & Coefficient & $\begin{array}{l}\text { Écart } \\
\text { type }\end{array}$ \\
\hline \multicolumn{11}{|c|}{$\begin{array}{l}\text { Nombre de changements de catégorie socioprofessionnelle (à } 2 \text { chiffres) } \\
\text { depuis le premier emploi (réf.: aucun): }\end{array}$} \\
\hline Un & $-0,08$ & 0,05 & 0,04 & 0,06 & 0,27 & 0,15 & 0,14 & 0,12 & $0,34 * *$ & 0,13 \\
\hline Deux & 0,05 & 0,06 & $0,36 * * *$ & 0,01 & 0,25 & 0,18 & 0,02 & 0,17 & $0,42 *$ & 0,18 \\
\hline Trois et plus & $0,51 * * *$ & 0,07 & $0,61 * * *$ & 0,11 & $0,68 * * *$ & 0,19 & $0,54 * *$ & 0,18 & $0,69 * *$ & 0,21 \\
\hline $\begin{array}{l}\text { A connu du chômage dans } \\
\text { le passé (réf.: N'a jamais } \\
\text { connu de période de } \\
\text { chômage) }\end{array}$ & 0,11 & 0,08 & $-0,01$ & 0,10 & $1,15 * * *$ & 0,14 & $0,85 * * *$ & 0,13 & $-0,13$ & 0,14 \\
\hline Nombre d'observations & \multirow{2}{*}{\multicolumn{2}{|c|}{$\begin{array}{l}45549 \\
65,9 \%\end{array}$}} & \multirow{2}{*}{\multicolumn{2}{|c|}{$\begin{array}{l}38173 \\
64,5 \%\end{array}$}} & \multirow{2}{*}{\multicolumn{2}{|c|}{$\begin{array}{l}45870 \\
73,6 \%\end{array}$}} & \multirow{2}{*}{\multicolumn{2}{|c|}{$\begin{array}{l}38594 \\
70,6 \%\end{array}$}} & \multirow{2}{*}{\multicolumn{2}{|c|}{$\begin{array}{l}2125 \\
69,3 \%\end{array}$}} \\
\hline $\begin{array}{l}\text { Pourcentage de paires } \\
\text { concordantes }\end{array}$ & & & & & & & & & & \\
\hline
\end{tabular}

Mêmes modèles que dans le tableau 2.

$* * *$ : significatif à $\mathbf{0 , 1} \% ; * *$ : significatif à $\mathbf{1} \% ; *$ : significatif à $\mathbf{5} \%$; coefficient en italique: significatif à $10 \%$.

réf: : modalité de référence.

Source: Enquête Histoire de vie, Insee, 2003.

Il s'agissait ici de répondre à la question d'une hausse des mobilités dans les carrières, idée qui sous-tend les débats sur la flexicurité mais qui reste discutée empiriquement. À partir des données originales de l'enquête Histoire de vie qui permettent d'observer des évolutions sur très longues périodes en retraçant les carrières sur le marché du travail français de trois générations d'individus, nous apportons une réponse mitigée.

Les données brutes montrent bien une hausse de la mobilité de génération en génération mais le contrôle des variations conjoncturelles, des effets de composition de la population active et du type de carrière passée en termes de mobilité, relativise fortement l'effet de la variable croisée "génération $\mathrm{x}$ âge». En particulier, alors que l'on peut reprocher à une enquête biographique de sous estimer les mobilités éloignées dans le passé, c'est plutôt une stabilité, voire une diminution pour les hommes, des changements professionnels en début de carrière (entre 20 et 29 ans) que l'on observe. En revanche, les transitions emploi-chômage ont incontestable- ment augmenté pour toutes les générations avec la dégradation du marché du travail, mais plus particulièrement chez les jeunes et les seniors. Les mobilités ne semblent donc pas augmenter uniformément avec le temps au sein de toutes les carrières. Il est certain que le risque de mobilité est plus grand quand il y a déjà eu mobilité ou chômage, ce qui révèle la persistance de la segmentation du marché du travail (RAmaux, 2006). Il est alors tentant de conclure à une stabilité des mobilités au sein de l'emploi (les changements professionnels, parmi lesquels se trouvent plus vraisemblablement les transitions choisies) accompagnée d'une hausse des mobilités entre emploi et chômage (révélant une précarisation accrue des emplois), hausse qui n'est pas uniforme au sein des carrières. On rejoint là des résultats déjà montrés à partir des données $\mathrm{FQP}$ ou de l'Enquête Emploi (GERME, 2001). Néanmoins, une telle conclusion reste à consolider, en prolongeant le présent travail par une étude plus fine des types de carrières professionnelles en termes de mobilité et de leur transformation. 


\section{Bibliographie}

Amossé T., (2002), «Vingt-cinq ans de transformation des mobilités sur le marché du travail», in Insee, Données Sociales, édition 2002-2003.

Amossé T., (2003), «Interne ou externe, deux visages de la mobilité professionnelle», Insee Première $\mathrm{n}^{\circ}$ 921, septembre.

Auriat N., (1997), Les défaillances de la mémoire humaine. Aspects cognitifs des enquêtes rétrospectives, PUF Ined.

Behaghel L., (2003), «Insécurité de l'emploi: le rôle protecteur de l'ancienneté a-t-il baissé en France?», Économie et Statistique, no 366, décembre, p. 3-29.

Burnod G., Chenu A., (2001), «Employés qualifiés et non qualifiés: une proposition d'aménagement de la nomenclature des catégories socioprofessionnelles », Travail et Emploi, no 86, p. 87-105.

Cahuc P., Kramarz F., (2004), De la précarité à la mobilité vers une sécurité sociale professionnelle, La Documentation française, Paris.

Cerc, (2005), La sécurité de l'emploi face aux défis des transformations économiques, Rapport $\mathrm{n}^{\circ} 5$, La Documentation Française, Paris.

CGT, (2006), «Communication de J.-P. Gabriel», Séminaire MESPI (Mutations de l'État dans la société post-industrielle), Paris, 30 mai.

Chapoulie S., (2000), «Une nouvelle carte de la mobilité professionnelle», Économie et Statistique, no 331.

Conseil européen, (2003), «Décision du Conseil du 22 juillet 2003, relative aux lignes directrices pour les politiques de l'emploi des États membres» (2003/578/ CE), Journal officiel de l'Union européenne, L 197, 5 août.

Courgeau D., Lelièvre E., (1990), «L'approche biographique en démographie», Revue Française de Sociologie, XXXI, 1.

Coutrot L., Dubar C., (1992), Cheminements professionnels et mobilités sociales, La Documentation française, Paris, 1992.

Crenner E., Donnat O., Guérin-Pace F., Houseaux F., VIlle I., (2006), «L'élaboration d'une enquête quantitative sur la construction des identités », Économie et Statistiques, no 393-394.

Delarre S., Duhautois R., (2004), «La mobilité intragroupe des salariés : le poids de la proximité géographique et structurale», Économie et Statistique, no 369-370.
Dupray A., (2005), "Les mobilités en début de vie professionnelle. Externes ou internes aux entreprises, des évolutions aux effets très différents ", Cereq-Bref, nº 216 , février.

FougÈRE D., (2003), «Instabilité de l'emploi et précarisation des trajectoires», in ANPE Actes des troisièmes entretiens de l'Emploi, p. 105-110.

Germe J.-F., (2001), «Au-delà des marchés internes: quelles mobilités, quelles trajectoires?», Formation Emploi, $\mathrm{n}^{0} 76$.

Givord P., Maurin E., (2003), «La montée de l'instabilité professionnelle et ses causes», Revue économique, vol. $54, \mathrm{n}^{\circ} 3$, p. 617-626.

Goux D., (1991), «Coup de frein sur les carrières», Économie et Statistique, $\mathrm{n}^{\circ} 249$, décembre.

Goux D., (2003), «Une histoire de l'Enquête Emploi», Économie et Statistique, no 362.

Granovetter M., (2000), Le marché autrement. Les réseaux dans l'économie, Desclée de Brouwer, coll. «Sociologie économique», Paris.

Heckman J., Borjas J., (1980), «Does Unemployment Cause Future Unemployment? Definitions, Questions and Answers form a Continuous Time Model of Heterogeneity and State Dependence», Economica, vol. $47, n^{\circ} 187$.

InSEE, (2006), «Enquête sur la formation et la qualification professionnelle (FQP)», Sources et méthodes, note méthodologique du 10/11. http://www.insee.fr/fr/nom def_met/sources/pdf/FQP.pdf

L'HorTy Y., (2004), «Instabilité de l'emploi: quelles ruptures de tendance?», Les Papiers du Cerc, no 200401 , février.

Leclair M., Roux S., (2005), «Mesure et utilisation des emplois instables dans les entreprises», Actes des Journées de méthodologie statistique.

Marchal E., Torny D., (2003), «Des petites aux grandes annonces: évolution du marché des offres d'emploi (1960-2000)», Travail et Emploi, no 95.

OCDE, (2004), «Réglementation relative à la protection de l'emploi et performance du marché du travail», Perspectives de l'Emploi de l'OCDE.

Ramaux C., (2006), Emploi: éloge de la stabilité, Essai Mille et une nuits, Paris. 


\section{Annexe \\ Descriptif par génération de l'échantillon des individus ayant au moins un an d'expérience professionnelle en France (en \%)}

Comme il s'agit de données rétrospectives collectées en 2003, les générations n'ont pas connu les mêmes durées moyennes de carrière; c'est pourquoi, dans le tableau suivant, le nombre de catégories socioprofessionnelles connues et le pourcentage d'individus ayant connu au moins un épisode de chômage ne sont pas directement comparables d'une génération à l'autre.

\begin{tabular}{|c|c|c|c|}
\hline Générations : & 1940-49 & 1950-59 & 1960-69 \\
\hline Effectifs: & 1375 & 1670 & 1463 \\
\hline Femmes & 49,2 & 53,4 & 53,5 \\
\hline Hommes & 50,8 & 46,6 & 46,5 \\
\hline Immigrés & 14,7 & 13,4 & 10,2 \\
\hline \multicolumn{4}{|l|}{ Niveau d'études atteint (en 2003): } \\
\hline Aucune étude à dernière année d'études primaires & 32,1 & 16,6 & 3,7 \\
\hline Premier cycle d'enseignement général & 10,8 & 12,6 & 9,9 \\
\hline Deuxième cycle d'enseignement général & 10,3 & 11,7 & 34,4 \\
\hline Enseignement technique ou professionnel court & 22,6 & 31,0 & 36,8 \\
\hline Enseignement technique ou professionnel long & 6,0 & 6,5 & 8,0 \\
\hline Enseignement supérieur & 18,3 & 21,6 & 29,5 \\
\hline Âge moyen lors du premier emploi: & 19,6 ans & 20,2 ans & 20,7 ans \\
\hline \multicolumn{4}{|l|}{ Catégorie socioprofessionnelle du premier emploi: } \\
\hline Agriculteurs & 4,0 & 1,9 & 1,3 \\
\hline Artisans, commerçants et chefs d'entreprise & 1,4 & 1,3 & 1,1 \\
\hline Cadres & 6,6 & 4,9 & 7,7 \\
\hline Professions intermédiaires & 14,9 & 15,4 & 19,6 \\
\hline Employés non qualifiés & 12,7 & 14,3 & 13,2 \\
\hline Employés qualifiés et de la Fonction publique & 17,8 & 21,6 & 20,4 \\
\hline Ouvriers non qualifiés & 23,1 & 22,1 & 18,1 \\
\hline Ouvriers qualifiés & 12,9 & 14,0 & 12,1 \\
\hline Autre & 2,6 & 1,1 & 1,0 \\
\hline \multicolumn{4}{|l|}{$\begin{array}{l}\text { Nombre total de catégories socioprofessionnelles } \\
\text { connues dans la carrière (en 2003): }\end{array}$} \\
\hline 1 & 26,2 & 31,2 & 34,8 \\
\hline 2 & 33,8 & 32,3 & 32,7 \\
\hline 3 & 20,7 & 20,7 & 19,8 \\
\hline$>4$ & 19,3 & 15,8 & 12,7 \\
\hline Pourcentage à avoir connu au moins un épisode de chômage: & 18,7 & 20,8 & 25,6 \\
\hline \multicolumn{4}{|l|}{ Situation en 2003: } \\
\hline En emploi & 50,4 & 80,8 & 83,2 \\
\hline Au chômage & 7,1 & 7,0 & 7,7 \\
\hline En retraite & 27,4 & 1,4 & 0,1 \\
\hline En inactivité (sauf retraite) & 15,1 & 10,8 & 9,0 \\
\hline
\end{tabular}

Source: Enquête Histoire de vie, Insee, 2003. 